\title{
Taxonomy and phylogeny of the Absidia (Cunninghamellaceae, Mucorales) introducing nine new species and two new combinations from China
}

Xiao-Yong Liu ( $\square$ liuxiaoyong@im.ac.cn )

Shandong Normal University

Research

Keywords: Mucoromycota, New taxa, Classification, Morphology, Molecular phylogeny

Posted Date: August 18th, 2021

DOl: https://doi.org/10.21203/rs.3.rs-820672/v1

License: (c) (1) This work is licensed under a Creative Commons Attribution 4.0 International License.

Read Full License 


\title{
Taxonomy and phylogeny of the Absidia (Cunninghamellaceae, Mucorales) introducing nine new species and two new combinations from China
}

Tong-Kai Zong ${ }^{1,2}$, Heng Zhao ${ }^{3,4}$, Xiao-Ling Liu ${ }^{3,5}$, Li-Ying Ren ${ }^{6}$, Chang-Lin Zhao ${ }^{2,7}$, Xiao-Yong $\mathrm{Liu}^{1,3, *}$

${ }^{1}$ College of Life Sciences, Shandong Normal University, Jinan 250014, China

${ }^{2}$ Key Laboratory for Forest Resources Conservation and Utilization in the Southwest Mountains of China, Ministry of Education, Southwest Forestry University, Kunming 650224, China

${ }^{3}$ State Key Laboratory of Mycology, Institute of Microbiology, Chinese Academy of Sciences, Beijing 100101, China

${ }^{4}$ Institute of Microbiology, School of Ecology and Nature Conservation, Beijing Forestry University, Beijing 100083, China.

${ }^{5}$ College of Life Science, University of Chinese Academy of Sciences, Beijing 100049, China.

${ }^{6}$ College of Plant Protection, Jilin Agricultural University, Changchun 130118, China. ${ }^{7}$ College of Biodiversity Conservation, Southwest Forestry University, Kunming 650224, China.

*Correspondence: liuxiaoyong@im.ac.cn

\begin{abstract}
Absidia is ubiquitous and plays an important role in medicine and biotechnology. In the present study, nine new species were described from China in the genus Absidia, i.e. $A$. ampullacea, A. brunnea, A. chinensis, A. cinerea, A. digitata, A. oblongispora, A. sympodialis, $A$. varians, and $A$. virescens. Besides, two varieties $A$. cylindrospora var. nigra and $A$. spinosa var. biappendiculata were elevated to a specific rank as A. nigra comb. nov. and $A$. biappendiculata comb. nov., respectively. These new taxa were
\end{abstract}


proposed based on a comprehensive investigation of morphological traits (such as shape and size of sporangia, sporangiospores and projections on columellae), physiological feature (maximum growth temperatures), and multi-locus sequences (including internal transcribed spacer, large subunit D1-D2 domains of nuclear ribosomal DNA, partial translation elongation factor 1 alpha gene and actin gene). All species mentioned above are illustrated, and an identification key to all the known species of Absidia in China is included.

KEYWORDS: Mucoromycota, New taxa, Classification, Morphology, Molecular phylogeny

\section{INTRODUCTION}

The genus Absidia Tiegh. (Cunninghamellaceae, Mucorales) was established by van Tieghem (1876). Absidia members are ubiquitous, mostly from soil and sometimes also associated with animals and plants. For example, the ex-type of A. spinosa var. biappendiculata Rall \& Solheim was isolated from the leaves of Comandra pallida (Rall and Solheim 1964), and the ex-type strains of A. californica J.J. Ellis \& Hesselt. and A. stercoraria Hyang B. Lee, H.S. Lee and T.T.T. Nguyen both live on rat dung (Ellis and Hesseltine 1965; Li et al. 2016). Although several species are vital causative agents of mucormycosis, some species are capable of producing chitin, chitosan, chitooligosaccharides (Kaczmarek et al. 2019) and hydrocortisone (Chen et al. 2020). This genus is characterized by rhizoids, stolons bearing single or a bunch of sporangiophores, a septum close to the top of sporangiophores, pyriform apophysate deliquescent-walled sporangia, distinct apophyses, and typically appendaged zygospores (Hoffmann et al. 2007; Hoffmann 2010).

The circumscription and classification of the Absidia have long been disputed. Before 1964, seven generic names allied to Absidia were successively proposed according to morphology, Tieghemella Berl. \& De Toni, Mycocladus Beauverie, Proabsidia Vuill., Lichtheimia Vuill., Pseudoabsidia Bainier, Protoabsidia Naumov 
and Gongronella Ribaldi (Berlese and de Toni 1888; Beauverie 1900; Vuillemin 1903a, 1903b; Bainier 1903; Naumov 1935; Ribaldi 1952). However, after a study on hundreds of strains comprising these genera, Hesseltine and Ellis (1964) retained Gongronella and Absidia only. The Gongronella has a globose apophysis with a constriction showing the attachment of the sporangial wall. They further divided Absidia into two subgenera, namely subg. Absidia and subg. Mycocladus (Beauverie) Hesselt. \& J.J. Ellis. The former produces appendages from the sespensors to envelop zygospores, whereas the latter does not produce any appendages. This classification framework was followed by Schipper (1990) who in turn recognized six groups in the subg. Absidia. However, with the advent of DNA-based molecular phylogenetics, the taxonomic classification of the genus has been refined. Hawksworth et al. (1995) did not accept the above morphologybased subgeneric delimitation, but synonymized all these allied genera with Absidia instead.

Recently, a comprehensive study of molecular phylogenetics, morphology and physiology has provided a more reliable delimitation among Absidia species (Hoffmann et al. 2007), where Absidia was classified into three groups: 1) the thermotolerant species with an optimal growth temperature of $37-45{ }^{\circ} \mathrm{C}$;2) the mesophilic species with an optimal growth temperature of $25-34{ }^{\circ} \mathrm{C}$, which have been accepted up to now as Absidia sensu stricto; and 3) the mycoparasitic species, potential to parasitise other mucoralean hosts. Soon, the thermotolerant species were transferred into the genus Lichtheimia (Hoffmann et al. 2009a); and the mycoparasitic species were placed into a new genus Lentamyces Kerst. Hoffm. \& K. Voigt (Hoffmann and Voigt 2008).

Currently, 39 species have been reported worldwide in Absidia (Bainier 1889; Hesseltine and Ellis 1961, 1964, 1966; Ellis and Hesseltine 1965, 1966; Index Fungorum http://www.indexfungorum.org, accessed on June 18, 2021). Among these species, 20 were proposed before 2001. Taxonomical research in Absidia seems to have stalled for a long time between 2002 and 2014. Since 2015, as many as 19 new species have been explosively described thanks to the strategy of combining morphology, physiology and phylogeny: A. caatinguensis D.X. Lima and A.L. Santiago, A. cornuta 
D.X. Lima, C.A. de Souza, H.B. Lee and A.L. Santiago, A. jindoensis Hyang B. Lee and T.T.T. Nguyen, A. koreana Hyang B. Lee, Hye W. Lee and T.T. Nguyen, $A$. multispora T.R.L. Cordeiro, D.X. Lima, Hyang B. Lee and A.L. Santiago, A. panacisoli T. Yuan Zhang, Ying Yu, He Zhu, S.Z. Yang, T.M. Yang, Meng Y. Zhang and Yi X. Zhang, A. pararepens Jurjević, M. Kolařík and Hubka, A. pernambucoensis D.X. Lima, C.M. Souza-Motta and A.L. Santiago, A. saloaensis T.R.L. Cordeiro, D.X. Lima, Hyang B. Lee and A.L. Santiago, A. stercoraria, A. terrestris Rosas de Paz, Dania García, Guarro, Cano and Stchigel, A. bonitoensis C.L. Lima, D.X. Lima, Hyang B. Lee and A.L. Santiago, A. ovalispora H. Zhao and X.Y. Liu, A. soli V.G. Hurdeal, E. Gentekaki, Hyang B. Lee \& K.D. Hyde, A. edaphica V.G. Hurdeal, E. Gentekaki, Hyang B. Lee \& K.D. Hyde, A. globospora T.K. Zong \& X.Y. Liu, A. medulla T.K. Zong \& X.Y. Liu, A. turgida T.K. Zong \& X.Y. Liu and A. zonata T.K. Zong \& X.Y. Liu. (Ariyawansa et al. 2015; Li et al. 2016; Crous et al. 2018, 2020; Wanasinghe et al. 2018; Zhang et al. 2018; Cordeiro et al. 2020; Lima et al. 2020; de Lima et al. 2021; Hurdeal et al. 2021; Zhao et al. 2021; Zong et al. 2021). Totally, 13 species are distributed in China (Zheng and Liu 2018; Zhang et al. 2018; Zhao et al. 2021; Zong et al. 2021).

Recently, 16 strains of Absidia were isolated from China but could not be assigned to any described species. Morphological, physiological and molecular data (multi-gene locus included ITS, D1-D2 domains of LSU rDNA, TEF-1 $\alpha$ and Act) are presented herein to support them within the Absidia sensu stricto, including nine new species and two new combinations. In addition, the synoptic key to all the 23 known species of Absidia in China is revised.

\section{MATERIALS AND METHODS}

\section{Isolation and Strains}

Strains were isolated from the soil collected in China about 20 years ago or even earlier. Approximately $1 \mathrm{~g}$ of soil samples was suspended in $100 \mathrm{~mL}$ sterilized water and shaken vigorously. Then, $100 \mu \mathrm{L}$ of the suspension was added onto a potato dextrose agar (PDA: $20 \mathrm{~g} / \mathrm{L}$ glucose, $20 \mathrm{~g} / \mathrm{L}$ agar, $200 \mathrm{~g} / \mathrm{L}$ potato, and $1000 \mathrm{~mL}$ distilled water) 
with $100 \mathrm{mg} / \mathrm{mL}$ each of streptomycin sulfate and ampicillin. The plate was incubated upside down at $27{ }^{\circ} \mathrm{C}$ and examined once a day with a stereomicroscope (SMZ1500, Nikon Corporation, Japan). Upon the presence of cultures, a single colony was picked onto a new PDA plate. Living cultures were deposited in the China General Microbiological Culture Collection Center, Beijing, China (CGMCC). Dried cultures were preserved in the Herbarium Mycologicum Academia Sinica, Beijing, China (HMAS).

\section{Morphology and Growth Experiments}

Pure strains were cultivated with malt extract agar (MEA: $20 \mathrm{~g} / \mathrm{L}$ malt extract, $20 \mathrm{~g} / \mathrm{L}$ agar, and $1000 \mathrm{~mL}$ distilled water). For morphological observation, they were incubated at $27{ }^{\circ} \mathrm{C}$ for $4-7 \mathrm{~d}$ and daily examined under a microscope (Axio Imager A2, Carl Zeiss Microscopy, Germany). For determining maximum growth temperatures, they were initially incubated at $32{ }^{\circ} \mathrm{C}$ for $4 \mathrm{~d}$, and then at higher temperatures until the colonies stopped growing. The color code of colonies was determined with the chromatography of Ridgway (1912).

\section{DNA Extraction, PCR Amplification and Sequencing}

Mycelia were grown at $27{ }^{\circ} \mathrm{C}$ for $5 \mathrm{~d}$ on PDA plates, and then cell DNAs were extracted with GO-GPLF-400 kit (GeneOnBio Corporation, Changchun, China). A span including the internal transcribed spacer (ITS) and large subunit (LSU) D1-D2 domain of rDNA were amplified with primer pair NS5M and LR5M (Wang et al. 2014). The partial translation elongation factor 1 alpha gene (TEF-1 $\alpha)$ was amplified with primer pair EF1-983F (Rehner and Buckley 2005) and TEF1LLErev (Jaklitsch et al. 2005). The partial actin gene (Act) was amplified with primer pair Act-1 and Act-4R (Voigt and Wostemeyer 2000). The PCR procedure for all these gene loci was as follows: an initial temperature at $95{ }^{\circ} \mathrm{C}$ for $5 \mathrm{~min}(\mathrm{rDNA}$ and TEF-1 a ) or $3 \mathrm{~min}(\mathrm{Act})$, then 30 cycles of denaturation at $95{ }^{\circ} \mathrm{C}$ for $30 \mathrm{~s}$ (rDNA and TEF-1 $\alpha$ ) or $60 \mathrm{~s}(A c t)$, annealing at $55{ }^{\circ} \mathrm{C}$ for $60 \mathrm{~s}$ and extension at $72{ }^{\circ} \mathrm{C}$ for $60 \mathrm{~s}$, and finally an extra extension at 
$72{ }^{\circ} \mathrm{C}$ for $10 \mathrm{~min}$. PCR products were purified and sequenced at BGI Tech Solutions Beijing Liuhe Co., Limited, Beijing, China. All newly generated sequences were deposited at GenBank (Table 1).

\section{Phylogenetic Analyses}

The software platform Geneious 9.0.2 (http://www.geneious.com) was used to assemble and proofread DNA sequences. All the sequences were realigned using AliView version 3.0 (Larsson 2014). The sequence alignments and phylogenetic trees were deposited at TreeBase (submission ID 28429). Sequences of Cunninghamella elegans and C. blakesleeana retrieved from GenBank were used as outgroups in multigene analyses following Hoffmann et al. (2007).

Phylogenetic analyses were carried out using algorithms Maximum Parsimony (MP), Maximum Likelihood (ML) and Bayesian Inference (BI). MP phylogenetic analyses followed Zhao and $\mathrm{Wu}$ (2017), and the tree construction was performed with PAUP* version 4.0b10 (Swofford 2002). All characters were equally weighted and gaps were treated as missing data. Trees were inferred using a heuristic search with TBR branch swapping and 1000 random sequence additions. Max-trees were set to 5000, branches of zero length were collapsed and all parsimonious trees were saved. Clade robustness was assessed using a bootstrap analysis with 1,000 replicates (Felsenstein 1985). Descriptive tree statistics tree length (TL), consistency index (CI), retention index (RI), rescaled consistency index (RC), and homoplasy index (HI) were calculated for each maximum parsimonious tree generated.

ML phylogenetic analyses were conducted with raxmlGUI 2.0 beta (Edler et al. 2020). A general time reversible model was used with a gamma-distributed rate variation $(\mathrm{GTR}+\mathrm{G})$ and 1000 bootstrap replicates were carried out.

BI phylogenetic analyses were calculated with MrBayes 3.2.7a (Ronquist et al. 2012) by a general time reversible model with an estimate of the proportion of invariant sites and a gamma distribution for variable rates across sites $(\mathrm{GTR}+\mathrm{I}+\mathrm{G})$. Four Markov chains were run simultaneously from random starting tree for 2,300,000 generations and the tree was sampled every 100 generations. The chains stopped once the average 
standard deviation of split frequencies decreased lower than 0.01 . The first one-fourth of generations were discarded as burn-in. A majority rule consensus tree of all remaining trees was calculated. Branches were considered as significantly supported if they received Maximum Likelihood bootstrap (MLB) $\geqslant 70 \%$, Maximum Parsimony bootstrap $(\mathrm{MPB}) \geqslant 70 \%$, or Bayesian posterior probabilities $(\mathrm{BPP}) \geqslant 0.95$ (Hillis and Bull 1993; Huelsenbeck and Hillis 1993; Leaché and Reeder 2002).

\section{RESULTS}

\section{Phylogenetic Analyses}

The multi-gene dataset included sequences from 64 strains representing 44 species of Absidia and two outgroups. The dataset had an aligned length of 3402 characters, of which 1865 characters were constant, 370 were variable and parsimony-uninformative, and 1167 were variable and parsimony-informative. MP analyses yielded 135 equally parsimonious trees $(\mathrm{TL}=8091, \mathrm{CI}=0.3297, \mathrm{HI}=0.6703, \mathrm{RI}=0.5653, \mathrm{RC}=0.1864)$. At the end of the inference, the average standard deviation of split frequencies was 0.009960 (BI). All BI, ML and MP phylogenetic trees resulted in similar topologies and consequently were integrated (Fig. 1).

\section{Taxonomic Treatments}

Absidia ampullacea T.K. Zong \& X.Y. Liu, sp. nov. (Fig. 2)

MycoBank no.: XXXXXX (to add after review).

Holotype: China, Beijing $\left(40^{\circ} 3^{\prime} 49^{\prime \prime} \mathrm{N}, 116^{\circ} 1^{\prime} 26^{\prime \prime} \mathrm{E}\right)$, from soil sample, 31 December 2019, Liu Xiaoyong (HMAS 350295, ex-type CGMCC 3.16054).

Etymology: ampullacea (Lat.) referring to the ampulliform-shaped swollen hyphae and sporangiophores.

Description: Colonies on MEA, irregularly zonate, attaining $46 \mathrm{~mm}$ diameter after $5 \mathrm{~d}$ at $27{ }^{\circ} \mathrm{C}$, white at first and then Saccardo's olive (R16). Hyphae hyaline at first, becoming brown when mature, sometimes ampulliform-shaped swollen, 6.0-13.0(16.5) $\mu \mathrm{m}$ wide. Stolons branched, hyaline, smooth, septate, 4.0-9.0 $\mu \mathrm{m}$ wide. Rhizoids 
finger-like, mostly twice or more repeatedly, with a septum at the base. Sporangiophores erect or slightly bent, 1-6 in whorls, unbranched, simple, monopodial or sympodial, hyaline, with a septum $14.5-21.5 \mu \mathrm{m}$ below apophyses, sometimes a swelling beneath sporangia, 30.0-320.0 $\times 2.5-5.5 \mu \mathrm{m}$. Apophyses distinct, bell-shaped, slightly pigmented, $4.0-9.5 \mu \mathrm{m}$ high, $2.5-6.0(-8.0) \mu \mathrm{m}$ wide at the base, and 9.5-18.0(20.0) $\mu \mathrm{m}$ wide at the top. Sporangia globose to pyriform, multispored, deliquescentwalled, 17.5-37.5 × 17.5-45.0 $\mu \mathrm{m}$. Columellae hemispherical, hyaline, smooth, occasionally with a $0.5-2.0 \mu \mathrm{m}$ verrucous projection at the apex, (8.5-)10.0-22.5 $\times$ (10.5-)14.0-24.0 $\mu \mathrm{m}$. Collars distinct. Sporangiospores oval to subglobose, 3.0-4.0($4.5) \times 2.5-3.5(-4.0) \mu \mathrm{m}$. Zygospores not observed. Chlamydospores absent. No growth at $30{ }^{\circ} \mathrm{C}$.

Absidia brunnea T.K. Zong \& X.Y. Liu, sp. nov. (Fig. 3)

MycoBank no.: XXXXXX (to add after review).

Holotype: China, Qinghai Province, Xining, Huangzhong County, Ta'er Monastery, from soil sample, 2 August 1999, Wang Long (HMAS 350296, ex-type CGMCC 3.16055).

Etymology: brunnea (Lat.) referring to the brown colony on MEA.

Description: Colonies on MEA, obversely regularly concentric ring zonate, attaining $47 \mathrm{~mm}$ diameter after $5 \mathrm{~d}$ at $27{ }^{\circ} \mathrm{C}$, white at first and then sayal brown to snuff brown (R29). Hyphae hyaline at first, becoming brown when mature, 6.0-17.5 $\mu \mathrm{m}$ wide. Stolons branched, hyaline, smooth, septate, 3.5-10.0 $\mu \mathrm{m}$ wide. Rhizoids branch-shaped, weakly branched, with a septum at the base. Sporangiophores erect or slightly bent, 17 in whorls but mostly 4-6, unbranched, simple or monopodial, rarely sympodial, hyaline, with a septum 11.0-17.0 $\mu \mathrm{m}$ below apophyses, sometimes a swelling beneath sporangia, occasionally branched at the swelling, 50.0-320.0 $(-420) \times 3.0-5.0(-6.5) \mu \mathrm{m}$. Apophyses distinct, funnel-shaped, slightly pigmented, 3.0-10.0 $\mu \mathrm{m}$ high, $2.5-7.5 \mu \mathrm{m}$ wide at the base, and 10.0-17.5(-20.0) $\mu \mathrm{m}$ wide at the top. Sporangia globose to pyriform, multispored, deliquescent-walled, 17.5-31.0(-38.0) × 19.0-30.0(-34.5) $\mu \mathrm{m}$. Columellae hemispherical, hyaline, smooth, always with a 2.5-5.5(-6.0) $\mu \mathrm{m}$ clavate 
projection at the apex, 7.0-16.0(-18.5) $\times$ 10.5-24.0 $\mu \mathrm{m}$. Collars distinct. Sporangiospores two types, the smaller one cylindrical to oval, 3.0-4.0 $\times 2.0-2.5 \mu \mathrm{m}$, the larger one oval, with vacuole, 4.0-6.5(-7.0) $\times 3.0-5.0 \mu \mathrm{m}$, hyaline, smooth. Zygospores not observed. Chlamydospores absent. No growth at $35{ }^{\circ} \mathrm{C}$.

\section{Absidia chinensis T.K. Zong \& X.Y. Liu, sp. nov. (Fig. 4)}

MycoBank no.: XXXXXX (to add after review).

Holotype: China, Yunnan Province, Xishuangbanna Dai Autonomous Prefecture, Mengla County, Xishuangbanna Tropical Botanical Garden, from soil sample, 4 July 1994, Chen Guiqing (HMAS 350297, ex-type CGMCC 3.16056).

Paratype: China, Sichuan Province, Tibetan Qiang Autonomous Prefecture of Ngawa, Jiuzhaigou County, Jiuzhaigou Valley Scenic and Historic Interest Area, from soil sample, 23 May 1994, Chen Guiqing (HMAS 350298, ex-type CGMCC 3.16057).

Etymology: chinensis (Lat.) referring to the country where the ex-type and exparatype strains both were isolated.

Description: Colonies on MEA, obversely regularly concentric ring zonate, attaining $50 \mathrm{~mm}$ diameter after $5 \mathrm{~d}$ at $27{ }^{\circ} \mathrm{C}$, white at first and then drab gray (R46) to snuff brown (R29). Hyphae hyaline at first, becoming brown when mature, generally swollen, (6.0-)7.5-14.0(-15.5) $\mu \mathrm{m}$ wide. Stolons branched, hyaline, smooth, septate, 3.5-9.0(-11.0) $\mu \mathrm{m}$ wide. Rhizoids coralliform, mostly simple or two branched, with a septum at the base. Sporangiophores erect or slightly bent, 1-5 in whorls, mostly unbranched or simple, rarely monopodial, sympodial not observed, hyaline, with a septum 13.0-17.5(-22.0) $\mu \mathrm{m}$ below apophyses, generally a swelling beneath sporangia, sometimes branched at the swelling, 45.0-220.0 × (2.5-)3.0-5.0(-6.0) $\mu \mathrm{m}$. Apophyses distinct, bell-shaped or hourglass-shaped, slightly pigmented, 3.5-6.5(-7.0) $\mu \mathrm{m}$ high, 2.5-5.5 $\mu \mathrm{m}$ wide at the base, and (6.0-)7.0-13.0(-15.5) $\mu \mathrm{m}$ wide at the top. Sporangia globose to pyriform, multispored, deliquescent-walled, 15.0-28.0(-39.5) $\times 15.0-28.5(-$ 37.5) $\mu \mathrm{m}$. Columellae spherical or hemispherical, hyaline, smooth, always with a 2.0 $5.5 \mu \mathrm{m}$ papillary projection at the apex, $(5.0-) 6.0-14.0(-15.5) \times(7.5-) 9.0-16.5(-17.5)$ $\mu \mathrm{m}$. Collars present or absent, but distinct if present. Sporangiospores cylindrical to 
oval, hyaline, smooth, 3.5-4.5 × 2.0-2.5 $\mu \mathrm{m}$. Zygospores globose, brown to dark brown, rough, $(37.0-) 45.0-80.0(-90.0) \times(36.0-) 50.0-80.0 \mu \mathrm{m}$. Suspensor unequal, parallel or nearly so, brown, the larger one $27.0-44.0 \mu \mathrm{m}$ diameter, the smaller one mostly 20.0 $33.0 \mu \mathrm{m}$ diameter. Chlamydospores absent. No growth at $31{ }^{\circ} \mathrm{C}$.

Absidia cinerea T.K. Zong \& X.Y. Liu, sp. nov. (Fig. 5)

MycoBank no.: XXXXXX (to add after review).

Holotype: China, Beijing $\left(40^{\circ} 21^{\prime} 30^{\prime \prime} \mathrm{N}, 116^{\circ} 6^{\prime} 27^{\prime \prime} \mathrm{E}\right)$, from soil sample, 31 December 2019, Liu Xiaoyong (HMAS 350303, ex-type CGMCC 3.16062).

Etymology: cinerea (Lat.) referring to the gray color of colonies on MEA.

Description: Colonies on MEA, regularly concentric ring zonate, attaining $44 \mathrm{~mm}$ diameter after $5 \mathrm{~d}$ at $27{ }^{\circ} \mathrm{C}$, white at first and then pale mouse gray (R51). Hyphae hyaline at first, becoming brown when mature, 5.5-14.5 $\mu \mathrm{m}$ wide. Stolons branched, hyaline, smooth, septate, 3.5-7.0 $\mu \mathrm{m}$ wide. Rhizoids branch-shaped and taper at the end, mostly two branches arising at a place, with a septum at the base. Sporangiophores erect or slightly bent, 1-7 in whorls, unbranched, simple or monopodial arising from stolons, mostly sympodial arising from aerial mycelium, hyaline, with a septum 11.0$17.0 \mu \mathrm{m}$ below apophyses, $50.0-150.0 \times 2.5-5.0 \mu \mathrm{m}$. Apophyses distinct, bell-shaped or funnel-shaped, slightly pigmented, $2.0-5.5(-7.5) \mu \mathrm{m}$ high, $2.5-5.5 \mu \mathrm{m}$ wide at the base, and 6.0-12.5 $\mu \mathrm{m}$ wide at the top. Sporangia globose to pyriform, multispored, deliquescent-walled, (7.0-)11.0-22.0(-27.5) $\times(7.0-) 13.0-25.0 \mu \mathrm{m}$. Columellae hemispherical, hyaline, smooth, always with a 1.3-5.0 $\mu \mathrm{m}$ rod-shaped or needle-like projection at the apex, $(5.0-) 5.5-12.0(-14.0) \times 7.0-15.0 \mu \mathrm{m}$. Collars present or absent, but distinct if present. Sporangiospores cylindrical, slightly constricted in the center, hyaline, smooth, 4.5-6.0 × 2.0-2.5 $\mu \mathrm{m}$. Zygospores globose, brown to dark brown, rough, 36.0-73.0(-81.0) $\mu \mathrm{m}$ diameter. Suspensor mostly one, rarely unequal two, brown, with 8-13 appendages on single suspensor, 17.0-33.0 $\mu \mathrm{m}$ diameter. Chlamydospores absent. No growth at $35^{\circ} \mathrm{C}$.

Absidia digitata T.K. Zong \& X.Y. Liu, sp. nov. (Fig. 6) 
MycoBank no.: XXXXXX (to add after review).

Holotype: China, Xinjiang Uygur Autonomous Region, Ili Kazak Autonomous Prefecture, Qapqal Xibe Autonomous County, Wusun Mountain, from soil sample, 14 June 2002, Wang Xuewei (HMAS 350299, ex-type CGMCC 3.16058).

Etymology: digitata (Lat.) referring to the finger-like shape of rhizoids.

Description: Colonies on MEA, regularly flower-shaped zonate, attaining $66 \mathrm{~mm}$ diameter after $6 \mathrm{~d}$ at $27{ }^{\circ} \mathrm{C}$, white at first and then snuff brown (R29). Hyphae hyaline at first, becoming brown when mature, sometimes swollen, 8.0-18.0 $\mu \mathrm{m}$ wide. Stolons branched, hyaline or light brown, smooth, septate, 4.0-8.0(-9.5) $\mu \mathrm{m}$ wide. Rhizoids finger-like, mostly twice or more repeatedly, with a septum at the base. Sporangiophores erect or slightly bent, 1-6 in whorls, unbranched, generally simple each sporangiophore in whorls, rarely monopodial or sympodial, hyaline or light brown, with a septum 14.5-23.5(-25.5) $\mu \mathrm{m}$ below apophyses, occasionally a swelling beneath sporangia, $(60.0-) 80.0-350.0(-470.0) \times 3.5-7.0(-8.5) \mu \mathrm{m}$. Apophyses distinct, slightly pigmented, (5.5-)7.0-13.5 $\mu \mathrm{m}$ high, 4.5-7.5(-8.5) $\mu \mathrm{m}$ wide at the base, and 12.519.0(-21.0) $\mu \mathrm{m}$ wide at the top. Sporangia globose to pyriform, multispored, deliquescent-walled, $(24.0-) 27.0-47.0(-64.0) \times 26.5-43.0(-48.5) \mu \mathrm{m}$. Columellae hemispherical, hyaline, smooth, sometimes with a 4.0-9.0 $\mu \mathrm{m}$ clavate projection at the apex, occasionally with two projections, slightly bulbous at the end, 10.5-22.0 $\times$ (11.5-)18.0-30.0 $\mu \mathrm{m}$. Collars distinct if present. Sporangiospores globose, hyaline, smooth, non-uniform, 3.0-5.0 $\mu \mathrm{m}$ diameter. Zygospores not observed. Chlamydospores absent. No growth at $32{ }^{\circ} \mathrm{C}$.

Absidia oblongispora T.K. Zong \& X.Y. Liu, sp. nov. (Fig. 7)

MycoBank no.: XXXXXX (to add after review).

Holotype: China, Yunnan Province, Xishuangbanna Dai Autonomous Prefecture, Mengla County, Xishuangbanna Tropical Botanical Garden, from soil sample, 4 July 1994, Chen Guiqing (HMAS 350302, ex-type CGMCC 3.16061).

Etymology: oblongispora (Lat.) referring to the oblong sporangiospores.

Description: Colonies on MEA, slightly irregular concentric ring zonate, attaining 
$44 \mathrm{~mm}$ diameter after $5 \mathrm{~d}$ at $27{ }^{\circ} \mathrm{C}$, white at first and then pale mouse gray (R51) to light drab (R46). Hyphae hyaline at first, becoming brown when mature, (5.5-)7.09.5(-11.5) $\mu \mathrm{m}$ wide. Stolons branched, hyaline, smooth, with few septa near the base of sporangiophores, 4.0-8.0(-9.5) $\mu \mathrm{m}$ wide. Rhizoids root-like, branched repeatedly, with a septum at the base. Sporangiophores erect or slightly bent, 1-5 in whorls, mostly unbranched, rarely simple or monopodial, sympodial not observed, hyaline, with a septum (9.5-)11.5-16.0 $\mu \mathrm{m}$ below apophyses, occasionally with a septum at the base, $(33.0-) 75.0-250.0(-300.0) \times(3.0-) 3.5-5.5 \mu \mathrm{m}$. Apophyses distinct, slightly pigmented, 3.5-6.5 $\mu \mathrm{m}$ high, 3.5-6.5(-7.5) $\mu \mathrm{m}$ wide at the base, and 7.0-16.5(-19.0) $\mu \mathrm{m}$ wide at the top. Sporangia globose, multispored, deliquescent-walled, (13.5-)19.5-29.5 $\times$ (14.5-)19.0-31.0 $\mu \mathrm{m}$. Columellae mostly conical, rarely hemispherical, hyaline, smooth, always with a 3.0-7.5 $\mu \mathrm{m}$ bulbous projection at the apex, $(7.0-) 8.5-13.5(-15.0)$ $\times(8.5-) 10.5-16.5 \mu \mathrm{m}$. Collars present or absent, but distinct if present. Sporangiospores cylindrical to oblong, hyaline, smooth, uniform, 3.5-4.5 × 2.5-3.0 $\mu \mathrm{m}$. Zygospores not observed. Chlamydospores absent. No growth at $32{ }^{\circ} \mathrm{C}$.

\section{Absidia sympodialis T.K. Zong \& X.Y. Liu, sp. nov. (Fig. 8)}

MycoBank no.: XXXXXX (to add after review).

Holotype: China, Shaanxi Province, Hanzhong, Foping County, from soil sample, 13 October 2002, Wang Xuewei (HMAS 350304, ex-type CGMCC 3.16064).

Paratype: China, Beijing $\left(40^{\circ} 1^{\prime} 32^{\prime \prime} \mathrm{N}, 116^{\circ} 8^{\prime} 54^{\prime \prime} \mathrm{E}\right)$, from soil sample, 31 December 2019, Liu Xiaoyong (HMAS 350305, ex-type CGMCC 3.16063).

Etymology: sympodialis (Lat.) referring to the sympodial sporangiophores.

Description: Colonies on MEA, regularly zonate, attaining $76 \mathrm{~mm}$ diameter after $5 \mathrm{~d}$ at $27{ }^{\circ} \mathrm{C}$, white at first and cedar green (R6). Hyphae hyaline at first, becoming light brown, (9.0-)10.5-19.0 $\mu \mathrm{m}$ wide. Stolons branched, hyaline, smooth, with few septa near the base of sporangiophores, 6.5-12.5 $\mu \mathrm{m}$ wide. Rhizoids fibrous-rootshaped, taper at the end, branched mostly repeatedly, with a septum at the base. Sporangiophores erect or slightly bent, 1-6 in whorls, unbranched, simple, monopodial or sympodial, hyaline or light brown, with a septum (7.5-)9.0-15.5(-17.5) $\mu \mathrm{m}$ below 
apophyses, $(90.0-) 180.0-520.0(-630.0) \times 4.5-9.0(-10.5) \mu \mathrm{m}$. Apophyses distinct, slightly pigmented, (6.0-)7.0-12.5(-13.7) $\mu \mathrm{m}$ high, 6.0-12.0(-14.1) $\mu \mathrm{m}$ wide at the base, and (12.0-)14.0-28.0(-31.0) $\mu \mathrm{m}$ wide at the top. Sporangia globose to pyriform, multispored, deliquescent-walled, (20.0-)23.5-55.0(-63.0) × (18.5-)22.5-53.0(-63.0) $\mu \mathrm{m}$. Columellae spherical, oval to hemispherical or chestnut-shaped, hyaline, smooth, sometimes with a $2.0-4.0 \mu \mathrm{m}$ papillary or pointed projection at the apex, (8.0-)10.5$30.5(-40.0) \times(9.5-) 13.5-35.0(-39.0) \mu \mathrm{m}$. Collars present or absent, but distinct if present. Sporangiospores globose, hyaline, smooth, uniform, 2.5-3.5 $\mu \mathrm{m}$ diameter. Zygospores not observed. Chlamydospores absent. No growth at $33{ }^{\circ} \mathrm{C}$.

Absidia varians T.K. Zong, H. Zhao \& X.Y. Liu, sp. nov. (Fig. 9)

MycoBank no.: XXXXXX (to add after review).

Holotype: China, Yunnan Province, Dehong Dai and Jingpo Autonomous

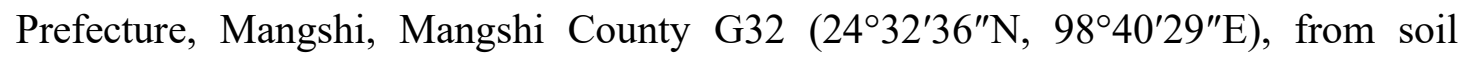
sample, 30 April 2021, Zhao Heng (HMAS 350306, ex-type CGMCC 3.16065).

Etymology: varians (Lat.) referring to the various shape of projections on columellae.

Description: Colonies on MEA, regularly zonate, attaining $67 \mathrm{~mm}$ diameter after $4 \mathrm{~d}$ at $27{ }^{\circ} \mathrm{C}$, white at first and then pale green-blue gray (R48). Hyphae hyaline to slightly pigmented, $8.5-12.0 \mu \mathrm{m}$ wide. Stolons branched, hyaline, smooth, septate, 5.0 $8.5 \mu \mathrm{m}$ wide. Rhizoids root-like, multi-branched, no septa. Sporangiophores erect or slightly bent, 1-4 in whorls but mostly 2-3 borne from stolons, unbranched, simple, monopodial or sympodial, hyaline, with a septum 13.5-19.0 $\mu \mathrm{m}$ below apophyses, 100.0-380.0(-480.0) × 4.5-9.0 $\mu \mathrm{m}$. Apophyses distinct, 6.0-12.0 $\mu \mathrm{m}$ high, 5.5-8.5(11.0) $\mu \mathrm{m}$ wide at the base, and 10.5-22.5 $\mu \mathrm{m}$ wide at the top. Sporangia subglobose to elliptical, multispored, deliquescent-walled, 26.0-50.0(-58.0) $\times 22.5-45.0(-50.0) \mu \mathrm{m}$. Columellae spherical or hemispherical, hyaline, smooth, always with all kinds of projections at the apex, mostly one, occasionally two, bulbous swelling, papillary, clavate or spinous, 11.0-26.5(-30.5) $\times 12.5-30.0(-35.0) \mu \mathrm{m}$. Collars distinct if present. Sporangiospores globose to oval, hyaline, smooth, 3.0-3.5(-4.0) $\times 2.0-3.0 \mu \mathrm{m}$. 
Zygospores not observed. Chlamydospores absent. No growth at $29{ }^{\circ} \mathrm{C}$.

Absidia virescens T.K. Zong \& X.Y. Liu, sp. nov. (Fig. 10)

MycoBank no.: XXXXXX (will add it after review).

Holotype: China, Yunnan Province, Xishuangbanna Dai Autonomous Prefecture, Jinghong, Mengyang Town, Xishuangbanna National Nature Reserve, from soil sample, 9 July 1994, Chen Guiqing (HMAS 350307, ex-type CGMCC 3.16067).

Paratype: China, Yunnan Province, Kumming, Yunnan Nationalities Village, from soil sample, 27 August 1995, Guo Yinglan (HMAS 350308, ex-type CGMCC 3.16066)

Etymology: virescens (Lat.) referring to the greenish colony on MEA.

Description: Colonies on MEA, regularly disc-shaped, attaining $63 \mathrm{~mm}$ diameter after $4 \mathrm{~d}$ at $27{ }^{\circ} \mathrm{C}$, white at first and then ring elm green (R17). Hyphae hyaline at first, becoming brown when mature, (6.0-)7.0-11.5 $\mu \mathrm{m}$ wide. Stolons branched, hyaline, smooth, with few septa near the base of sporangiophores, 4.5-9.0 $\mu \mathrm{m}$ wide. Rhizoids root-like, branched mostly twice or three times, with a septum at the base. Sporangiophores erect or slightly bent, 1-4 in whorls, mostly unbranched or simple, rarely monopodial or sympodial, hyaline, with a septum (10.0-)12.0-19.5(-22.5) $\mu \mathrm{m}$ below apophyses, occasionally with a septum at the base, sometimes a swelling beneath sporangia, $(75.0-) 120.0-350.0(-480.0) \times 5.5-10.5(-11.5) \mu \mathrm{m}$. Apophyses distinct, sometimes slightly pigmented, (5.5-)6.5-10.5(-14.0) $\mu \mathrm{m}$ high, 6.5-12.0(-15.5) $\mu \mathrm{m}$ wide at the base, and (12.5-)15.0-25.5 $\mu \mathrm{m}$ wide at the top. Sporangia globose to pyriform, multispored, deliquescent-walled, (15.5-)23.0-51.5 × (15.5-)20.5-45.5 $\mu \mathrm{m}$. Columellae spherical or oval to hemispherical, hyaline, smooth, sometimes with a single or two 3.0-6.5 $\mu \mathrm{m}$ projection at the apex, (10.5-)13.0-31.5(-40.5) $\times$ (11.5-)16.5-37.5(-45.0) $\mu \mathrm{m}$. Collars present or absent, but distinct if present. Sporangiospores globose to subglobose, hyaline, smooth, non-uniform, 3.0-5.0(-5.5) $\mu \mathrm{m}$ diameter, but mostly 3.0-3.5 $\mu \mathrm{m}$ diameter. Zygospores not observed. Chlamydospores absent. No growth at $32{ }^{\circ} \mathrm{C}$. 
Absidia nigra (Hesselt. \& J.J. Ellis) T.K. Zong \& X.Y. Liu, comb. nov. (Fig. 11)

MycoBank no.: XXXXXX (to add after review).

Basionym: Absidia cylindrospora var. nigra Hesselt. \& J.J. Ellis, Mycologia, 56(4), 595. 1964. [MB no.: 353238]

Holotype: United States, Wisconsin, near Jefferson Junction, from the soil of pastured hardwood sample, 1940 (type NRRL 3060, isotype CBS 127.68, isotype HMAS 350309,)

Additional strains: China, Jilin Province, Jiaohe, State-owned Forest Protection Center of Forestry Experimental Area of Jilin Province, from soil sample, 1 September 1991, Bai Fengyan (HMAS 350300, living culture CGMCC 3.16059); Inner Mongolia Autonomous Region, Hinggan League, Arxan, from soil sample, 16 August 1991, Bai Fengyan (HMAS 350301, living culture CGMCC 3.16060).

Etymology: nigra (Lat.) adopting from basionym.

Description: Colonies on MEA, regularly zonate, attaining $62 \mathrm{~mm}$ diameter after $6 \mathrm{~d}$ at $27{ }^{\circ} \mathrm{C}$, white at first and then light drab (R46). Hyphae hyaline at first, becoming brown when mature, sometimes swollen, 8.0-15.5(-18.5) $\mu \mathrm{m}$ wide. Stolons branched, hyaline, smooth, septate, 4.0-8.5 $\mu \mathrm{m}$ wide. Rhizoids finger-like, short, mostly twice or repeatedly, with a septum at the base. Sporangiophores erect or slightly bent, 1-5 in whorls, unbranched, simple, rarely monopodial or sympodial, hyaline, with a septum 11.5-21.0 $\mu \mathrm{m}$ below apophyses, sometimes a swelling beneath sporangia, 60.0-220.0($380.0) \times(2.5-) 3.0-6.5 \mu \mathrm{m}$. Apophyses distinct, slightly pigmented, 3.5-8.5 $\mu \mathrm{m}$ high, 3.3-5.9 $\mu \mathrm{m}$ wide at the base, and 9.5-18.5 $\mu \mathrm{m}$ wide at the top. Sporangia globose to pyriform, multispored, deliquescent-walled, (17.0-) 18.0-38.0(-46.5) × 16.5-38.5 $\mu \mathrm{m}$. Columellae spherical or oval to hemispherical, hyaline, smooth, sometimes with a 1.5$5.0 \mu \mathrm{m}$ clavate or slightly swollen projection at the apex, 8.5-19.0 $\times(9.5-) 11.5-23.0$ $\mu \mathrm{m}$. Collars distinct if present. Sporangiospores two types, cylindrical or globose, hyaline, smooth, 3.0-4.0 × 2.0-2.5 $\mu \mathrm{m}$ when cylindrical, $4.5-8.0(-9.0) \times 4.0-8.0 \mu \mathrm{m}$ when globose. Zygospores not observed. Chlamydospores absent. No growth at $31{ }^{\circ} \mathrm{C}$.

Absidia biappendiculata (Rall \& Solheim) T.K. Zong \& X.Y. Liu, comb. nov. 
(Fig. 12)

MycoBank no.: XXXXXX (to add after review).

Basionym: Absidia spinosa var. biappendiculata Rall \& Solheim, Mycologia, 56(1), 99. 1964. [MB no.: 348993]

Holotype: United States, Wyoming, Albany County, Medicine Bow Mountains, Dry Park near Fox Park, from the leaves of Comandra pallida, August 1960, G. Rall (NRRL 3033, isotype CBS 187.64, isotype HMAS 350310)

Etymology: biappendiculata (Lat.) adopting from basionym.

Description: Colonies on MEA, regularly zonate, attaining $75 \mathrm{~mm}$ diameter after $5 \mathrm{~d}$ at $27{ }^{\circ} \mathrm{C}$, white at first and then pale mouse gray (R51). Hyphae hyaline to slightly pigmented, 5.5-11.0 $\mu \mathrm{m}$ wide. Stolons branched, hyaline, smooth, septate, 3.5-5.5 $\mu \mathrm{m}$ wide. Rhizoids root-like, taper at the end, with a septum at the base. Sporangiophores erect or slightly bent, 1-5 in whorls, unbranched or simple, monopodial and sympodial not observed, hyaline, with a septum 11.0-17.0 $\mu \mathrm{m}$ below apophyses, 65.0-180.0($210.0) \times 2.5-5.0 \mu \mathrm{m}$. Apophyses distinct, slightly pigmented, 3.5-7.0(-9.0) $\mu \mathrm{m}$ high, 2.5-4.5 $\mu \mathrm{m}$ wide at the base, and 8.0-14.0(-20.0) $\mu \mathrm{m}$ wide at the top. Sporangia globose to pyriform, multispored, deliquescent-walled, (16.0-)19.0-29.0 $\times$ (15.5-)18.5-27.0 $\mu \mathrm{m}$. Columellae hemispherical to subglobose, hyaline, smooth, sometimes with a 3.0-7.0 $\mu \mathrm{m}$ spinous projection at the apex, occasionally two projections, or with a conical projection in some of smaller columellae, 7.5-15.0(-19.0) $\times 8.5-16.0(-20.0) \mu \mathrm{m}$. Collars distinct if present. Sporangiospores cylindrical to oval, slightly constricted in the center, hyaline, smooth, (3.5-)4.0-4.5 $\times 2.0-2.5 \mu \mathrm{m}$. Zygospores globose, brown to dark brown, rough, 33.0-66.0 $\mu \mathrm{m}$ diameter. Suspensor mostly two, nearly equal, parallel or nearly so, sometimes only one, hyaline or brown, 14.0-24.0(-27.0) $\mu \mathrm{m}$ diameter. Chlamydospores absent. No growth at $35^{\circ} \mathrm{C}$.

\section{DISCUSSION}

Phylogenetically, the multi-gene tree (Fig. 1) shows that nine new species and two new combinations all cluster in Absidia. Among these species, Absidia ampullacea is a sister group of A. caatinguensis with a 92/ - /1.00 support value. However, A. caatinguensis 
physiologically differs from $A$. ampullacea in maximum growth temperature $\left(35{ }^{\circ} \mathrm{C}\right.$ vs. $30{ }^{\circ} \mathrm{C}$ ). They also morphologically differ in shape and size of sporangiospores and projection on columellae. In detail, cylindrical sporangiospores $(5.0-7.0 \times 2.5-3.7 \mu \mathrm{m})$ and bulbous projection (up to $5.7 \mu \mathrm{m}$ in length) of $A$. caatingguensis are both longer than subglobose to oval sporangiospores $(3.0-4.5 \times 2.5-4.0 \mu \mathrm{m})$ and verrucous projection (up to $2.0 \mu \mathrm{m}$ in length) of A. ampullacea (Ariyawansa et al. 2015).

Abisidia brunnea is most closely related to A. soli (100/100/1.00). However, physiologically, A. soli differs from $A$. brunnea in maximum growth temperature $\left(37{ }^{\circ} \mathrm{C}\right.$ vs. $35{ }^{\circ} \mathrm{C}$ ). Morphologically, A. soli is characterized by its sporangiophores up to 6 in whorls, septa distantly below apophyses $(21.5-37.5 \mu \mathrm{m})$, larger sporangia (16.0-51.0 $\times 15.0-45.5 \mu \mathrm{m}$, average $34.5 \times 30.0 \mu \mathrm{m})$, and sporangiospores only cylindrical and without vacuole (Hurdeal et al. 2021).

Absidia chinensis (100/100/1.00) clusters with A. nigra (100/100/1.00) but their sibling relationship does not receive a strong support. Physiologically, A. chinensis is similar to A. nigra in maximum growth temperature but morphologically they are different in sporangiospores, sporangiophores, rhizoids and apophyses. A. chinensis produces cylindrical sporangiospores only, swollen and branched sporangiophores, coralliform rhizoids and bell-shaped or hourglass-shaped apophyses, while $A$. nigra produces both clindrical and globose sporangiospores, swelling but never branched sporangiophores, relatively short rhizoids and never hourglass-shaped apophyses (Hesseltine and Ellis 1964).

Absidia cinerea appears to be phylogenetically basal to A. pseudocylindrospora Hesselt. \& J.J. Ellis and A. stercoraria, although with relatively low support values. However, physiologically, A. pseudocylindrospora have a maximum growth temperature of $37{ }^{\circ} \mathrm{C}$, but neither $A$. cinerea nor $A$. stercoraria can grow above $35{ }^{\circ} \mathrm{C}$. Morphologically, A. cinerea differs in that it produces longer cylindrical sporangiospores $(4.5-6.0 \times 2.0-2.5 \mu \mathrm{m})$ and branch-shaped rhizoids with septa, whereas A. pseudocylindrospora and A. stercoraria both bear shorter sporangiospores, $2.5 \times 3.5-5.0 \mu \mathrm{m}$ and $4.0-5.0 \times 2.0-3.0 \mu \mathrm{m}$, respectively. Rhizoids have rarely septa in A. pseudocylindrospora and are not well developed in A. stercoraria. Additionally, 
sporangiophores of $A$. cinerea arise in whorls of up to 7, whereas those of $A$. pseudocylindrospora and A. stercoraria arise in whorls of up to 11 and 5, respectively (Hesseltine and Ellis 1961; Li et al. 2016).

Absidia digitata is most closely related to A turgida with a high support value (99/100/1.00) and a similar maximum growth temperature $\left(32{ }^{\circ} \mathrm{C}\right.$ vs. $\left.33{ }^{\circ} \mathrm{C}\right)$. However, morphologically, A. turgida differs from A. digitata in that it produces sporangiophores singly or in whorls of up to 4, only one projection on the columellae, and such variable sporangiospores as globose, cylindrical or irregular (Zong et al. 2021).

Absidia oblongispora is most closely related to A. heterospora Y. Ling, which is strongly supported with a high value of $100 / 100 / 1.00$. At the same time, they have similar maximum growth temperatures physiologically $\left(32{ }^{\circ} \mathrm{C}\right.$ vs. $\left.31{ }^{\circ} \mathrm{C}\right)$. But morphologically, A. heterospora differs from A. oblongispora in that it produces larger columellae (10.5-34 $\mu \mathrm{m}$ diameter) without projection and two types of sporangiospores (3.8-7.7 $\mu \mathrm{m}$ when globose and 3.3-6.6 $\times 2.2-4.4 \mu \mathrm{m}$ when cylindrical to oval, Hesseltine and Ellis 1964), whereas A. oblongispora always presents projection on columellae and only smaller cylindrical to oval sporangiospores $(3.5-4.5 \times 2.5-3.0 \mu \mathrm{m})$.

Absidia sympodialis (100/100/1.00), A. virescens (100/100/1.00) and A. varians $(100 / 100 / 1.00)$ are nested in the globospora clade, and they all producing green colonies. A. sympodialis and A. virescens are sister taxa and cluster with $A$. globospora (100/100/1.00), having similar sporangia. Their relationship is highly supported by the multi-gene tree, with a $-/ 98 / 1.00$ support value. However, physiologically and morphologically, A. globospora is different by no growth at $29^{\circ} \mathrm{C}$, whereas the maximum growth temperature of $A$. sympodialis and $A$. virescens both are higher than $30{ }^{\circ} \mathrm{C}$. In addition, the sporangiophores of $A$. globospora are in whorls up to 5, whereas those of A. sympodialis and A. virescens arise in whorls of up to 6 and 4, respectively. A. virescens produces sporangiospores of non-uniform in size and columellae with two projections, some projection up to $6.5 \mu \mathrm{m}$ in length, whereas the sporangiospores of $A$. sympodialis and $A$. globospora are uniform in size, and both columellae with only one projection less than $5 \mu \mathrm{m}$ in length. A. sympodialis differs from $A$. virescens and $A$. globospora in the shape of rhizoids and columellae. It has fibrous-root-shaped rhizoids, 
differing from them in that both have root-like rhizoids. Additionally, columellae of $A$. sympodialis are hemispherical, oval and chestnut-shaped, unlike those hemispherical in A. virescens and A. globospora (Zong et al. 2021). Absidia varians (100/100/1.00) is phylogenetically next to A. glauca but morphologically similar to A. repens because they both have variable projections on columellae. A. repens is different from $A$. varians in that it having olive-gray colonies and two types of sporangiospores (Hesseltine and Ellis 1966).

Absidia nigra (100/100/1.00) and A. biappendiculata (100/100/1.00) were previously regarded as synonyms of A. cylindrospora Hagem and A. spinosa Lendn., respectively. These treatments of synonymization do not receive support either phylogenetically or morphologically. Phylogenetically, A. nigra is related to $A$. chinensis rather than $A$. cylindrospora, and A. biappendiculata clusters with $A$. stercoraria, A. pseudocylindrospora and A. cinerea (86/85/1.00) rather than A. spinosa. Morphologically, A. nigra differs from A. cylindrospora by not giving any mating reaction with any $A$. cylindrospora strains (Hesseltine and Ellis 1964). In addition, $A$. nigra produces cylindrical sporangiospores $3.0-4.0 \times 2.0-2.5 \mu \mathrm{m}$ and globose sporangiospores 4.5-8.0(-9.0) $\times$ 4.0-8.0 $\mu \mathrm{m}$, differing from $A$. cylindrospora which produces only cylindrical sporangiospores 3.3-5.5 × 2.2-3.5 $\mu \mathrm{m}$ (Hesseltine and Ellis 1964), as well as sporangiophores sometimes with a swelling beneath sporangia. $A$. biappendiculata is like A. spinosa in the appearance of sporangiospores, sporangia, and columellae (Rall et al. 1964). However, sporangiophores in A. spinosa are wider (5$10.5 \mu \mathrm{m}$ ) than those in A. biappendiculata (Rall et al. 1964). Additionally, A. spinosa produces only one projection on columellae (Hesseltine and Ellis 1964; Rall et al. 1964), unlike two projections in A. biappendiculata.

Physiologically, as mentioned in the introduction, growth temperature has been an important characteristic to distinguish Absidia s. str. (optimal growth temperature of 25-34 ${ }^{\circ} \mathrm{C}$ ) and Lichtheimia species (optimal growth temperature of 37-45 ${ }^{\circ} \mathrm{C}$, Hoffmann et al. 2007). Most species of Absidia s. str. were within the temperature range, but it was also stated that Absidia cuneospora G.F. Orr \& Plunkett, A. idahoensis var. thermophile G.Q. Chen \& R.Y. Zheng, A. koreana and A. pseudocylindrospora were 
able to grow above $37^{\circ} \mathrm{C}$ (Orr and Plunkett 1959; Hesseltine and Ellis 1961; Chen and Zheng 1998; Ariyawansa et al. 2015). On the other hand, Lichtheimia sphaerocystis Alastr.-Izq. \& Walther and L. brasiliensis A.L. Santiago, N. Lima \& R.J.V. Oliveira were limited in growth at $40{ }^{\circ} \mathrm{C}$ (Alastruey-Izquierdo et al. 2010; André et al. 2014). This indicates that growth temperature does not accurately distinguish the species of Absidia s. str. and Lichtheimia (syn. Absidia pro parte). The maximum growth temperatures of the nine new species reported here are $29-35^{\circ} \mathrm{C}$, falling in the temperature range of Absidia s. str.

Morphologically, Hoffmann et al. (2007) found that Absidia usually have projections on the apex of columellae. The nine new species and two combinations described here also have this characteristic. Therefore, we believe that projection on columellae is a typical feature of the genus Absidia, except for the species $A$. heterospora (Hesseltine and Ellis 1964).

The Absidia genus was proposed to be divided into several groups distinguishable by their sporangiospores (Kwaśna et al. 2006; Hoffmann et al. 2007, 2009b; Hoffmann and Voigt 2008; Hoffmann 2010). However, with new species of Absidia were increasingly described, some of them were found to have two and more shapes of sporangiospores, such as A. pararepens, A. repens, A. turgida, etc (Fig. 1, Crous et al. 2020; Cordeiro et al. 2020; Zong et al. 2021). Therefore, the clades on the phylogenetic tree should be further divided, especially the clade previously defined as cylindrical spores. Additionally, A. digitata does not goup within the globose spores clade, even though it has globose sporangiospores only, indicating that the shape of sporangiospores in Absidia might polyphyletic evolved.

Types in Absidia are mainly distributed in North America (e.g., Canada, Honduras, Mexico, United States), South America (e.g., Argentina, Brazil), Europe (e.g., Czech, Germany, Holland, United Kingdom, Switzerland), Africa (e.g., Tanzania) and Asia (e.g., China, India, South Korea, Thailand) (Fig. 13), but have never been reported in Oceania. Only type of Absidia idahoensis var. thermophila was from China before 1998 (Chen and Zheng 1998), suggesting an insufficient sampling by Chinese mycologists. In recent years, more and more new species, e.g. Absidia globospora, A. medulla, A. 
ovalispora, A. panacisoli, A. turgida and A. zonata, have gradually been reported from China (Zhang et al. 2018; Zhao et al. 2021; Zong et al. 2021). This study further supplements nine new species, making the list of Chinese Absidia types as long as 16.

As many as eleven new taxa are placed in Absidia, and therefore it is necessary to update the synoptic key for the 23 species distributed in China as follows.

Key to the known species of Absidia in China

1. Sporangiospores obviously two or more types.............................2

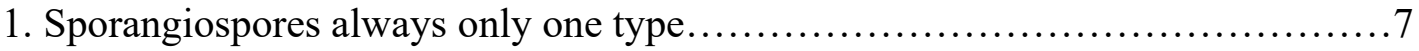

2. Sporangiospores sometimes irregular in shape............................ 3

2. Sporangiospores always regular in shape.................................5

3. With elliptical or very elongate sporangia; elliptical sporangia typically borne singly in great numbers along stolons ................................. repens

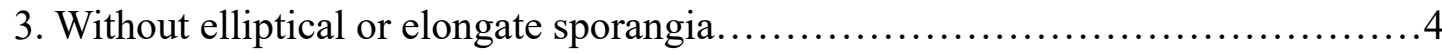

4. Hyphae without swelling, $<9 \mu \mathrm{m}$ in width; sporangiophores sometimes simple, more often monopodial or verticillate; columellae sometimes with a short projection A. idahoensis

4. Hyphae occasionally swelling, $>9 \mu \mathrm{m}$ in width; sporangiophores unbranched or sometimes simple; columellae always with a projection up to $9 \mu \mathrm{m}$ in length

A. turgida

5. Columellae without distinct apical projections A. heterospora

5. Columellae with apical projections 6

6. Sporangiophores 1-7 in whorls; rhizoids relatively longer; sporangiospores never globose, the larger one oval with vacuole; no growth at $35{ }^{\circ} \mathrm{C} \ldots \ldots \ldots \ldots . . . .$. . brunnea

6. Sporangiophores 1-5 in whorls; rhizoids relatively shorter; sporangiospores globose in the larger one, without vacuole; no growth at $31{ }^{\circ} \mathrm{C}$ A. nigra

7. Sporangiospores typically globose or nearly so........................... 8

7. Sporangiospores mostly cylindrical or oval............................... 13

8. Colonies brownish; rhizoids finger-like; with clavate projections on the columellae. A. digitata

8. Colonies greenish; rhizoids root-like or other shapes 9 
9. Sporangia subglobose to elliptical; projections on the columellae variable in shape. A. varians

9. Sporangia globose to pyriform; projections on the columellae invariable in shape.

10. Sporangiospores non-uniform in size; columellae sometimes with the single or two projections. A. virescens

10. Sporangiospores uniform in size; columellae with only one projection. .11

11. Columellae sometimes chestnut-shaped; rhizoids fibrous-root-shaped.... A. sympodialis

11. Columellae hemispherical but never chestnut-shaped; rhizoids root-like. 12

12. Sporangiophores not reaching $10 \mu \mathrm{m}$ in width; sporangia rarely reaching $55 \mu \mathrm{m}$ diam; no growth at $29{ }^{\circ} \mathrm{C}$ A. globospora

12. Sporangiophores reaching $12 \mu \mathrm{m}$ in width; sporangia mostly 50-60 $\mu \mathrm{m}$ diam; no growth at $37{ }^{\circ} \mathrm{C}$ A. glauca

13. Collar absent; sporangiospores ovoidal to ellipsoidal A. ovalispora

13. Collar always present; sporangiospores typically cylindrical 14

14. Sporangiophores never in pairs or in whorls. A. panacisoli

14. Sporangiophores in pairs and in whorls 15

15. Sporangiophores as many as $7-11$ in whorls. 16

15. Sporangiophores no more than 6 in whorls.

16. Rhizoids typically unseptate A. spinosa

16. Rhizoids septate. .17

17. Apophyses bell-shaped or funnel-shaped A. cinerea

17. Apophyses never bell-shaped or funnel-shaped. .18

18. Projections on columellae $<5 \mu \mathrm{m}$ in length, taper at the top. A. zonata

18. Projections on columellae $>5 \mu \mathrm{m}$ in length, rounded at the top. $A$. pseudocylindrospora

19. Maximum growth temperatures $>34{ }^{\circ} \mathrm{C}$ A. cylindrospora

19. Maximum growth temperatures $\leq 34{ }^{\circ} \mathrm{C}$ .20

20. Hyphae and sporangiophores both swollen.... .21 
20. Hyphae or sporangiophores never swollen .22

21. Sporangiospores cylindrical to oval; rhizoids coralliform; papillary projections on columellae $2-5.5 \mu \mathrm{m}$ in length.... A. chinensis

21. Sporangiospores oval to subglobose; rhizoids finger-like; verrucous projections on columellae up to $2 \mu \mathrm{m}$ in length A. ampullacea 22. Sporangiophores $1-5$ in whorls, no sympodial; columellae mostly conical, rarely hemispherical, with the bulbous projection up to $7.5 \mu \mathrm{m}$ in length.... A. oblongispora

22. Sporangiophores 1-6 in whorls, sympodial; columellae mostly hemispherical, with the pointed projection up to $4.5 \mu \mathrm{m}$ in length. A. medulla

\section{DECLARATIONS}

\section{Ethics approval and consent to participate}

Not applicable.

\section{Adherence to national and international regulations}

Not applicable.

\section{Consent for publication}

Not applicable.

\section{Availability of data and material}

Details of the availability of the data and materials used in this study can be found within the text. DNA sequences were submitted to GenBank [https://www.ncbi.nlm.nih.gov/]. Scientific name and morphological description deposited at MycoBank [https://www.mycobank.org]. Alignments were deposited at TreeBase (submission ID: 28429, https://www.treebase.org/treebase-web/). Living Strains were deposited in the China General Microbiological Culture Collection Center, Beijing, China (CGMCC, http://www.cgmcc.net/). Dried Strains were deposited in the Herbarium Mycologicum Academiae Sinicae, Beijing, China (HMAS). 


\section{Competing interests}

The authors declare no conflict of interest.

\section{Funding}

This research was funded by the National Natural Science Foundation of China, grant number 31970009 and the Yunnan Fundamental Research Project, Grant number 202001AS070043.

\section{Authors' contributions}

ZTK, ZCL and LXY contributed to conception and design of the study. ZTK wrote the draft of the manuscript. ZCL and LXY improved the manuscript. ZTK, ZH and LXY observed and described the morphology. LXL and RLY collected and analysed the molecular data. All authors have read and agreed to the published version of the manuscript.

\section{Acknowledgements}

We thank Fengyan Bai, Long Wang, Xuewei Wang, Guiqing Chen, Yinglan Guo (Institute of Microbiology, CAS) for collecting soil samples.

\section{References}

Alastruey-Izquierdo A, Hoffmann K, de Hoog GS, Rodriguez-Tudela JL, Voigt K, Bibashi E, Walther G (2010) Species recognition and clinical relevance of the zygomycetous genus Lichtheimia (syn. Absidia pro parte, Mycocladus). Journal of Clinical Microbiology 48: 2154-2170. doi: https://doi.org/10.1128/JCM.01744-09.

André LCMA, Hoffmann K, Lima DX, de Oliveira RJV, Vieira HEE, Malosso E, Maia ML, Silva GAA (2014) new species of Lichtheimia (Mucoromycotina, Mucorales) isolated from Brazilian soil. Mycological Progress 13: 343-352. doi: https://doi.org/10.1007/s11557-013-0920-8. 
Ariyawansa HA, Hyde KD, Jayasiri SC, Buyck B, Chethana KT, Dai DQ, Dai YC, Daranagama DA, Jayawardena RS, Lücking R, et al. (2015) Fungal diversity notes 111-252-taxonomic and phylogenetic contributions to fungal taxa. Fungal Diversity 75: 27-274. doi: 10.1007/s13225-015-0346-5.

Bainier G (1889) Sur l'Absidia coerulea. Bulletin de la Société Botanique de France 36: 184-184. doi: https://doi.org/10.1080/00378941.1889.10830441.

Bainier M (1903) Sur quelques espèces de Mucorinées nouvelles ou peu connues. Bulletin de la Société Mycologique de France 19: 153-172.

Beauverie J (1900) Mycocladus verticillatus (gen. nov. sp. nov.). Annales de l'Université de Lyon 3: 162-180.

Berlese AN, de Toni JB (1888) Phycomyceteae. Sylloge Fungorum 7: 181-322.

Chen GQ, Zheng RY (1998) A new thermophilic variety of Absidia idahoensis from China. Mycotaxon 69: 173-179.

Chen J, Fan F, Qu G, Tang J, Xi Y, Bi C, Sun Z, Zhang X (2020) Identification of Absidia orchidis steroid 11ß-hydroxylation system and its application in engineering Saccharomyces cerevisiae for one-step biotransformation to produce hydrocortisone. Metabolic Engineering 57: 31-42. doi: https://doi.org/10.1016/j.ymben.2019.10.006.

Crous PW, Luangsa-Ard JJ, Wingfield MJ, Carnegie AJ, Hernández-Restrepo M, Lombard L, Roux J, Barreto RW, Baseia IG, Cano-Lira JF, et al. (2018) Fungal Planet description sheets: 785-867. Persoonia: Molecular Phylogeny and Evolution of Fungi 41: 238. doi: https://doi.org/10.3767/persoonia.2018.41.12.

Cordeiro TRL, Nguyen TTT, Lima DX, Silva SBGD, Lima CFD, Leitão JD, Gurgel LMS, Lee HB, de Azevedo Santiago ALCM (2020) Two new species of the industrially relevant genus Absidia (Mucorales) from soil of the Brazilian Atlantic Forest. Acta Botanica Brasilica 34: 549-558. doi: https://doi.org/10.1590/0102$33062020 \mathrm{abb} 0040$.

Crous PW, Wingfield MJ, Chooi YH, Gilchrist CL, Lacey E, Pitt JI, Roets F, Swart WJ, Cano-Lira JF, Valenzuela-Lopez N, et al. (2020) Fungal Planet description sheets: 1042-1111. Persoonia: Molecular Phylogeny and Evolution of Fungi 44: 301. doi: https://doi.org/10.3767/persoonia.2020.44.11.

de Lima CLF, Lima DX, Cordeiro TRL, Lee HB, Nguyen TTT, Gurgel LMS, de Azevedo Santiago ALCM (2021) Absidia bonitoensis (Mucorales, Mucoromycota), a new species isolated from the soil of an upland Atlantic forest 
in Northeastern Brazil. Nova Hedwigia 112: 241-251. doi: 10.1127/nova_hedwigia/2021/0614.

Edler D, Klein J, Antonelli A, Silvestro D (2020) raxmlGUI 2.0: a graphical interface and toolkit for phylogenetic analyses using RAxML. Methods Ecology and Evolution. doi: https://doi.org/10.1101/800912.

Ellis JJ, Hesseltine CW (1965) The genus Absidia: globose-spored species. Mycologia 57: 222-235. doi: https://doi.org/10.1080/00275514.1965.12018205.

Ellis JJ, Hesseltine CW (1966) Species of Absidia with ovoid sporangiospores. II. Sabouraudia 5: 59-77. doi: https://doi.org/10.1080/00362176785190111.

Felsenstein J (1985) Confidence intervals on phylogenetics: an approach using bootstrap. Evolution 39: 783-791. doi: https://doi.org/10.1111/j.15585646.1985.tb00420.x.

Hawksworth DL, Kirk PM, Sutton BC, Pegler DN (1995) Ainsworth \& Bisby's Dictionary of the Fungi, 8th edn. CAB International, Egham.

Hesseltine CW, Ellis JJ (1961) Notes on Mucorales, especially Absidia. Mycologia 53: 406-426. doi: https://doi.org/10.1080/00275514.1961.12017970.

Hesseltine CW, Ellis JJ (1964) The genus Absidia: Gongronella and cylindrical-spored species of Absidia. Mycologia 56: 568-601. doi: https://doi.org/10.1080/00275514.1964.12018145.

Hesseltine CW, Ellis JJ (1966) Species of Absidia with ovoid sporangiospores. I. Mycologia 58: 761-785. doi: https://doi.org/10.1080/00275514.1966.12018369.

Hillis DM, Bull JJ (1993) An empirical test of bootstrapping as a method for assessing confidence in phylogenetic analysis. Systematic Biology 42: 182-192. doi: https://doi.org/10.1093/sysbio/42.2.182

Hoffmann K (2010) Identification of the Genus Absidia (Mucorales, Zygomycetes): A Comprehensive Taxonomic Revision. In: Gherbawy Y, Voigt K (eds) Molecular Identification of Fungi. Springer, Heidelberg. doi: https://doi.org/10.1007/978-3642-05042-8_19.

Hoffmann K, Voigt K (2008) Absidia parricida plays a dominant role in biotrophic fusion parasitism among mucoralean fungi (Zygomycetes): Lentamyces, a new genus for A. parricida and A. zychae. Plant Biology 11: 537-554. doi: https://doi.org/10.1111/j.1438-8677.2008.00145.x.

Hoffmann K, Discher S, Voigt K (2007) Revision of the genus Absidia (Mucorales, Zygomycetes): based on physiological, phylogenetic and morphological 
characters: thermotolerant Absidia spp. form a coherent group, the Mycocladiaceae fam. nov. Mycological Research 111: 1169-1183. doi: https://doi.org/10.1016/j.mycres.2007.07.002.

Hoffmann K, Walther G, Voigt K (2009) Mycocladus vs. Lichtheimia: a correction (Lichtheimiaceae fam. nov., Mucorales, Mucoromycotina). Mycological Research 113: 275-278.

Hoffmann K, Telle S, Walther G, Eckart M, Kirchmair M, Prillinger H, Prazenica A, Newcombe G, Dölz F, Papp T, et al. (2009) Diversity, genotypic identification, ultrastructural and phylogenetic characterization of zygomycetes from different ecological habitats and climatic regions: limitations and utility of nuclear ribosomal DNA barcode markers. In: Gherbawy Y, Mach R, Rai M (eds) Current Advances in Molecular Mycology. Nova Science Publishers, New York, pp 263312.

Huelsenbeck JP, Hillis DM (1993) Success of phylogenetic methods in the four-taxon case. Systematic Biology 42: 247-264. doi: https://doi.org/10.1093/sysbio/42.3.247.

Hurdeal VG, Gentekaki E, Lee HB, Jeewon R, Hyde KD, Tibpromma S, Mortimer PE, Xu J (2021) Mucoralean fungi in Thailand: novel species of Absidia from tropical forest soil. Cryptogamie Mycologie 42: 39-61. doi: https://doi.org/10.5252/cryptogamie-mycologie2021v42a4.

Jaklitsch WM, Komon M, Kubicek CP, Druzhinina IS (2005) Hypocrea voglmayrii sp. nov. from the Austrian Alps represents a new phylogenetic clade in Hypocrea/Trichoderma. Mycologia 97: 1365-1378. doi: https://doi.org/10.3852/mycologia.97.6.1365.

Kaczmarek MB, Struszczyk-Swita K, Li X, Szczęsna-Antczak M, Daroch M (2019) Enzymatic modifications of chitin, chitosan, and chitooligosaccharides. Frontiers in Bioengineering and Biotechnology 7: 243. doi: https://doi.org/10.3389/fbioe.2019.00243.

Kwaśna H, Elaine WARD, Bateman GL (2006) Phylogenetic relationships among Zygomycetes from soil based on ITS1/2 rDNA sequences. Mycological Research 110: 501-510. doi: https://doi.org/10.1016/j.mycres.2006.02.004.

Larsson A (2014) AliView: a fast and lightweight alignment viewer and editor for large datasets. Bioinformatics 30: 3276-3278. doi: https://doi.org/10.1093/bioinformatics/btu531. 
Leaché AD, Reeder TW (2002) Molecular systematics of the eastern fence lizard (Sceloporus undulatus): a comparison of parsimony, likelihood, and Bayesian approaches. Systematic Biology 51: 44-68. doi: https://doi.org/10.1080/106351502753475871.

Li GJ, Hyde KD, Zhao RL, Hongsanan S, Abdel-Aziz FA, Abdel-Wahab MA, Alvarado P, Alves-Silva G, Ammirati JF, Ariyawansa HA, et al. (2016) Fungal diversity notes 253-366: taxonomic and phylogenetic contributions to fungal taxa. Fungal Diversity 78: 1-237. doi: https://doi.org/10.1007/s13225-016-0366-9.

Lima DX, Cordeiro TR, de Souza CA, de Oliveira RJ, Lee HB, Souza-Motta CM, de Azevedo Santiago ALCM (2020) Morphological and molecular evidence for two new species of Absidia from Neotropic soil. Phytotaxa 446: 61-71. doi: https://doi.org/10.11646/phytotaxa.446.1.8.

Naumov NA (1935) Opredelitel Mukorovykh [Determination of Mucorales], 2nd edn. Botanical Institute Academy of Sciences USSR, Moscow.

Orr GF, Plunkett OA (1959) A new species of Absidia from California. Mycologia 51: 203-209. doi: https://doi.org/10.1080/00275514.1959.12024813.

Rall G, Solheim WG (1964) A variety of Absidia isolated from Comandra pallida. Mycologia 56: 99-102. doi: https://doi.org/10.1080/00275514.1964.12018086.

Rehner SA, Buckley E (2005) A Beauveria phylogeny inferred from nuclear ITS and EF1- $\alpha$ sequences: evidence for cryptic diversification and links to Cordyceps teleomorphs. $\quad$ Mycologia 97: 84-98. doi: https://doi.org/10.3852/mycologia.97.1.84.

Ribaldi MS (1952) Sopra un interessante Zigomicete terricolo: Gongronella urceolifera n. gen. et n. sp. Rivista di Biologia 44: 157-166.

Ridgway R (1912) Color Standards and Color Nomenclature. Ridgway, Washington, DC. doi: https://doi.org/10.5962/bhl.title.144788.

Ronquist F, Teslenko M, Van Der Mark P, Ayres DL, Darling A, Höhna S, Larget B, Liu L, Suchard MA, Huelsenbeck JP (2012) MrBayes 3.2: efficient Bayesian phylogenetic inference and model choice across a large model space. Systematic Biology 61: 539-542. doi: https://doi.org/10.1093/sysbio/sys029.

Schipper MAA (1990) Notes on Mucorales - I. Observations on Absidia. Persoonia 14: 133-149.

Swofford DL (2002) PAUP*: Phylogenetic Analysis Using Parsimony (*and Other Methods), Version 4.0b10. Sinauer Associates, Sunderland, MA. 
van Tieghem P (1876) Troisième mémoire sur les Mucorinées. Annales des Siences Naturelles Botanique 4: 312-399.

Voigt K, Wöstemeyer J (2000) Reliable amplification of actin genes facilitates deeplevel phylogeny. Microbiological Research 155: 179-195. doi: https://doi.org/10.1016/S0944-5013(00)80031-2.

Vuillemin P (1903) Importance taxonomique de l'appareil zygosporé des Mucorinées. Bulletin de la Société Mycologique de France 19: 106-118.

Vuillemin P (1903) Le genre Tieghemella et la série des Absidiées. Bulletin de la Société Mycologique de France 19: 119-127.

Wanasinghe DN, Phukhamsakda C, Hyde KD, Jeewon R, Lee HB, Jones EG, Tibpromma S, Tennakoon DS, Dissanayake AJ, Jayasiri SC, et al. (2018) Fungal diversity notes 709-839: taxonomic and phylogenetic contributions to fungal taxa with an emphasis on fungi on Rosaceae. Fungal Diversity 89: 1-236. doi: https://doi.org/10.1007/s13225-018-0395-7.

Wang YN, Liu XY, Zheng RY (2014) Umbelopsis changbaiensis sp. nov. from China and the typification of Mortierella vinacea. Mycological Progress 13: 657-669. doi: https://doi.org/10.1007/s11557-013-0948-9.

Zhang TY, Yu Y, Zhu H, Yang SZ, Yang TM, Zhang MY, Zhang YX (2018) Absidia panacisoli sp. nov., isolated from rhizosphere of Panax notoginseng. International Journal of Systematic and Evolutionary Microbiology 68: 2468-2472. doi: https://doi.org/10.1099/ijsem.0.002857.

Zhao CL, Wu ZQ (2017) Ceriporiopsis kunmingensis sp. nov. (Polyporales, Basidiomycota) evidenced by morphological characters and phylogenetic analysis. Mycological Progress 16: 93-100. doi: https://doi.org/10.1007/s11557-016-12598.

Zhao H, Zhu J, Zong TK, Liu XL, Ren LY, Lin Q, Qiao M, Nie Y, Zhang DZ, Liu XY (2021) Two New Species in the Family Cunninghamellaceae from China. Mycobiology 49: 142-150. doi: https://doi.org/10.1080/12298093.2021.1904555.

Zheng RY, Liu XY (2018) Species Catalogue of China, Volume 3. Fungi: Chtrid, Zygomycotan, Glomeromycotan Fungi. Science Press, Beijing.

Zong TK, Zhao H, Liu XL, Ren LY, Zhao CL, Liu XY (2021) Taxonomy and phylogeny of four new species in Absidia (Cunninghamellaceae, Mucorales) from China. Frontiers in Microbiology. (accepted) 


\section{FIGURE LEGENDS}

Fig. 1 The Maximum Parsimony strict consensus tree illustrating the phylogeny of nine new species and two new combinations of Absidia in Cunninghamellaceae based on multi-gene sequences. Cunninghamella elegans and C. blakesleeana serve as outgroups. Branches are labeled with Maximum Likelihood bootstrap values higher than $70 \%$, Maximum Parsimony bootstrap values higher than $70 \%$ and Bayesian posterior probabilities more than 0.95 . The lower left scale represents steps. Sporangiospore shapes are annotated.

Fig. 2 Absidia ampullacea CGMCC 3.16054. (A) obverse colony on MEA; (B) reverse colony on MEA; (C) sporangium; (D-F) columellae; (G-I) swelling on sporangiospohores and hyphae; $(\mathbf{J})$ sporangiospores; $(\mathbf{K})$ rhizoids. Scale bars: $(\mathbf{C}-\mathbf{F}, \mathbf{K})$ $10 \mu \mathrm{m}$; (G-I) $20 \mu \mathrm{m} ;$ (J) $5 \mu \mathrm{m}$.

Fig. 3 Absidia brunnea CGMCC 3.16055. (A) obverse colony on MEA; (B) reverse colony on MEA; (C) sporangium; (D, E) columellae; (F, G) swelling on sporangiospohores; (H) sporangiospores; (I) rhizoids. Scale bars: (C-G, I) $10 \mu \mathrm{m}$; (H) $5 \mu \mathrm{m}$.

Fig. 4 Absidia chinensis CGMCC 3.16056. (A) obverse colony on MEA; (B) reverse colony on MEA; (C) sporangium; (D-F) columellae; (G, H) swelling on sporangiospohores; (I) sporangiospores; (J) rhizoids; (K-N) zygospores. Scale bars: (C-F, J-N) $10 \mu \mathrm{m}$; (G, H) $20 \mu \mathrm{m}$; (I) $5 \mu \mathrm{m}$.

Fig. 5 Absidia cinerea CGMCC 3.16062. (A) obverse colony on MEA; (B) reverse colony on MEA; (C) sporangium; (D-F) columellae; $(\mathbf{G})$ sporangiospores; $(\mathbf{H})$ rhizoids (I) sporangiophores in whorls; (J-L) zygospores. Scale bars: (C-F) $10 \mu \mathrm{m}$; (G) $5 \mu \mathrm{m}$; (H-L) $20 \mu \mathrm{m}$.

Fig. 6 Absidia digitata CGMCC 3.16058. (A) obverse colony on MEA; (B) reverse 
colony on MEA; (C) sporangium; (D-G) columellae; (H) swelling on hyphae; (I) sporangiospores; (J) rhizoids. Scale bars: (C-G) $10 \mu \mathrm{m}$; (H, J) $20 \mu \mathrm{m}$; (I) $5 \mu \mathrm{m}$.

Fig. 7 Absidia oblongispora CGMCC 3.16061. (A) obverse colony on MEA; (B) reverse colony on MEA; (C) sporangium; (D-F) columellae; $(\mathbf{G})$ sporangiospores; $(\mathbf{H})$ rhizoids. Scale bars: (C-F, H) $10 \mu \mathrm{m}$; (G) $5 \mu \mathrm{m}$.

Fig. 8 Absidia sympodialis CGMCC 3.16064. (A) obverse colony on MEA; (B) reverse colony on MEA; $(\mathbf{C})$ sporangium; $(\mathbf{D}, \mathbf{E})$ columellae; $(\mathbf{F})$ sporangiospores; $(\mathbf{G}, \mathbf{H})$ rhizoids; (I) sympodial sporangiospores. Scale bars: (C-E, G-I) $20 \mu \mathrm{m}$; (F) $5 \mu \mathrm{m}$.

Fig. 9 Absidia varians CGMCC 3.16065. (A) obverse colony on MEA; (B) reverse colony on MEA; (C, D) sporangia; $(\mathbf{E}-\mathbf{L})$ columellae; (M) sporangiospores; $(\mathbf{N}, \mathbf{O})$ rhizoids. Scale bars: (C-L) $10 \mu \mathrm{m}$; (M) $5 \mu \mathrm{m}$; (N, O) $20 \mu \mathrm{m}$.

Fig. 10 Absidia virescens CGMCC 3.16067. (A) obverse colony on MEA; (B) reverse colony on MEA; (C) sporangium; (D-G) columellae; (H) swelling on sporangiospohores; (I) sporangiospores; (J, K) rhizoids; (L) monopodial sporangiospohores. Scale bars: (C-H, J-K) $20 \mu \mathrm{m}$; (I) $5 \mu \mathrm{m}$; (L) $50 \mu \mathrm{m}$.

Fig. 11 Absidia nigra CGMCC 3.16059. (A) obverse colony on MEA; (B) reverse colony on MEA; (C) sporangia; (D, E) columellae; (F) swelling on sporangiospohores; (G) sporangiospores; $(\mathbf{H})$ rhizoids (I, J) swollen hyphae. Scale bars: $(\mathbf{C}-\mathbf{F}, \mathbf{H}) 10 \mu \mathrm{m}$; (G) $5 \mu \mathrm{m} ;$ (I, J) $20 \mu \mathrm{m}$.

Fig. 12 Absidia biappendiculata CBS 187.64. (A) obverse colony on MEA; (B) reverse colony on MEA; (C) sporangium; (D-G) columellae; (H) sporangiospores; (I) rhizoids; (K-L) zygospores. Scale bars: (C-G) $10 \mu \mathrm{m}$; (H) $5 \mu \mathrm{m}$; (I-L) $20 \mu \mathrm{m}$.

Fig. 13 The geographic distribution of Absida types except for $A$. glauca and $A$. 
cylindrospora (neither is available). Triangles represent nine new species and two combinations proposed in this study. Circles represent previously reported species. 
Table 1 The list of species, strains, and GenBank accession numbers of sequences used in this study.

\begin{tabular}{|c|c|c|c|c|c|}
\hline \multirow{2}{*}{ Species } & \multirow{2}{*}{ Strains } & \multicolumn{4}{|c|}{ GenBank accession no. } \\
\hline & & ITS & LSU & TEF-1 $\alpha$ & Act \\
\hline Absidia ampullaceal & CGMCC $3.16054^{\mathrm{T}}$ & MZ354138 & MZ350132 & & \\
\hline A. anomala & CBS 125.68 & NR_103626 & NG_058562 & & \\
\hline A. anomala & FSU 5798 & EF030523 & & & EF030535 \\
\hline A. biappendiculata & CBS 187.64 ${ }^{\mathrm{T}}$ & MZ354153 & MZ350147 & MZ357420 & MZ357438 \\
\hline A. bonitoensis & URM 7889 & MN977786 & MN977805 & & \\
\hline A. brunnea & CGMCC $3.16055^{\mathrm{T}}$ & MZ354139 & MZ350133 & MZ357403 & MZ357421 \\
\hline A. caatinguensis & URM 7156 & КТ308169 & КТ308171 & & \\
\hline A. californica & CBS 314.78 & MH861141 & MH872902 & & \\
\hline A. californica & FSU 4747 & AY944872 & EU736300 & EU736246 & AY944758 \\
\hline A. californica & FSU 4748 & AY944873 & EU736301 & EU736247 & EU736224 \\
\hline A. chinensis & CGMCC $3.16056^{T}$ & MZ354140 & MZ350134 & & \\
\hline A. chinensis & CGMCC 3.16057 & MZ354141 & MZ350135 & & MZ357422 \\
\hline A. cinerea & CGMCC $3.16062^{T}$ & MZ354146 & MZ350140 & MZ357407 & $\operatorname{MZ357427}$ \\
\hline A. coerulea & CBS 101.36 & MH855718 & MH867230 & & \\
\hline
\end{tabular}




\begin{tabular}{|c|c|c|c|c|c|}
\hline A. coerulea & NRRL 1315 & AY944870 & AF113443 & AF157226 & AJ287133 \\
\hline A. cornuta & URM 6100 & MN625256 & MN625255 & & \\
\hline A. cuneospora & CBS 101.59 & NR_159602 & NG058559 & & \\
\hline A. cylindrospora & CBS 100.08 & JN205822 & JN206588 & & \\
\hline A. cylindrospora var. nigra & CBS 127.68 & & NG_058560 & & \\
\hline A. cylindrospora var. rhizomorpha & CBS 153.63 & & NG_058563 & & \\
\hline A. digitata & CGMCC $3.16058^{T}$ & MZ354142 & MZ350136 & MZ357404 & MZ357423 \\
\hline A. edaphica & MFLU 20-0416 & MT396372 & МT393987 & & MT410739 \\
\hline A. fusca & CBS 102.35 & NR103625 & NG058552 & & \\
\hline A. glauca & CBS 129233 & MH865253 & MH876693 & & \\
\hline A. glauca & CBS 101.08 & NR_111658 & NG_058550 & & \\
\hline A. glauca & FSU 660 & AY944879 & EU736302 & EU736248 & EU736225 \\
\hline A. globospora & CGMCC 3.16031 & MW671537 & MW671544 & MZ357412 & MZ357431 \\
\hline A. globospora & CGMCC 3.16035 & MW671538 & MW671545 & MZ357413 & MZ357432 \\
\hline A. globospora & CGMCC 3.16036 & MW671539 & MW671546 & MZ357414 & MZ357433 \\
\hline A. heterospora & SHTH021 & JN942683 & JN982936 & & \\
\hline A. jindoensis & CNUFC-PTI1-1 & MF926622 & MF926616 & MF926513 & MF926509 \\
\hline
\end{tabular}




\begin{tabular}{|c|c|c|c|c|c|}
\hline A. koreana & EML-IFS45-1 & KR030062 & KR030056 & KR030060 & KR030058 \\
\hline A. macrospora & FSU 4746 & AY944882 & EU736303 & EU736249 & AY944760 \\
\hline A. medulla & CGMCC 3.16034 & MW671542 & MW671549 & MZ357417 & MZ357436 \\
\hline A. medulla & CGMCC 3.16037 & MW671543 & MW671550 & MZ357418 & \\
\hline A. multispora & URM 8210 & MN953780 & MN953782 & & \\
\hline A. nigra & CGMCC $3.16059^{\mathrm{T}}$ & MZ354143 & MZ350137 & MZ357405 & MZ357424 \\
\hline A. nigra & CGMCC 3.16060 & MZ354144 & MZ350138 & MZ357406 & MZ357425 \\
\hline A. nigra & CBS 127.68 & MZ354152 & MZ350146 & MZ357419 & MZ357437 \\
\hline A. oblongispora & CGMCC $3.16061^{T}$ & MZ354145 & MZ350139 & & MZ357426 \\
\hline A. ovalispora & CGMCC 3.16018 & MW264071 & MW264130 & & \\
\hline A. panacisoli & SYPF 7183 & MF522181 & MF522180 & MF624251 & \\
\hline A. pararepens & CCF 6352 & MT193669 & MT192308 & & \\
\hline A. pernambucoensis & URM 7219 & MN635568 & MN635569 & & \\
\hline A. pseudocylindrospora & CBS 100.62 & NR_145276 & NG_058561 & & \\
\hline A. pseudocylindrospora & EML-FSDY6-2 & KU923817 & KU923814 & & KU923815 \\
\hline A. psychrophilia & FSU 4745 & AY944874 & EU736306 & EU736252 & AY944762 \\
\hline A. repens & CBS 115583 & NR103624 & HM849706 & & \\
\hline
\end{tabular}




\begin{tabular}{|c|c|c|c|c|c|}
\hline A. repens & NRRL 1336 & & AF113448 & AF157228 & AJ287136 \\
\hline A. saloaensis & URM 8209 & MN953781 & MN953783 & & \\
\hline A. soli & MFLU 20-0414 & MT396373 & MT393988 & & MT410740 \\
\hline A. spinosa & FSU 551 & AY944887 & EU736307 & EU736253 & EU736227 \\
\hline A. spinosa var. biappendiculata & CBS 187.64 & & MH870040 & & \\
\hline A. stercoraria & EML-DG8-1 & NR_148090 & KT921998 & KT922002 & KT922000 \\
\hline A. sympodialis & CGMCC $3.16064^{\mathrm{T}}$ & MZ354148 & MZ350142 & MZ357408 & \\
\hline A. sympodialis & CGMCC 3.16063 & MZ354147 & MZ350141 & & \\
\hline A. terrestris & FMR 14989 & LT795003 & LT795005 & & \\
\hline A. turgida & CGMCC 3.16032 & MW671540 & MW671547 & MZ357415 & MZ357434 \\
\hline A. varians & CGMCC $3.16065^{\mathrm{T}}$ & MZ354149 & MZ350143 & MZ357409 & MZ357428 \\
\hline A. virescens & CGMCC $3.16067^{T}$ & MZ354151 & MZ350145 & MZ357411 & MZ357430 \\
\hline A. virescens & CGMCC 3.16066 & MZ354150 & MZ350144 & MZ357410 & MZ357429 \\
\hline A. zonata & CGMCC 3.16033 & MW671541 & MW671548 & MZ357416 & MZ357435 \\
\hline Cunninghamella blakesleeana & CBS 782.68 & JN205869 & MH870950 & KJ156478 & \\
\hline Cunninghamella elegans & CBS 167.53 & JN205882 & HM849700 & KJ156494 & \\
\hline
\end{tabular}

*Sequences obtained herein are shown in bold. The " $\mathrm{T}$ " is abbreviated for ex-type strains. 


\section{Figures}

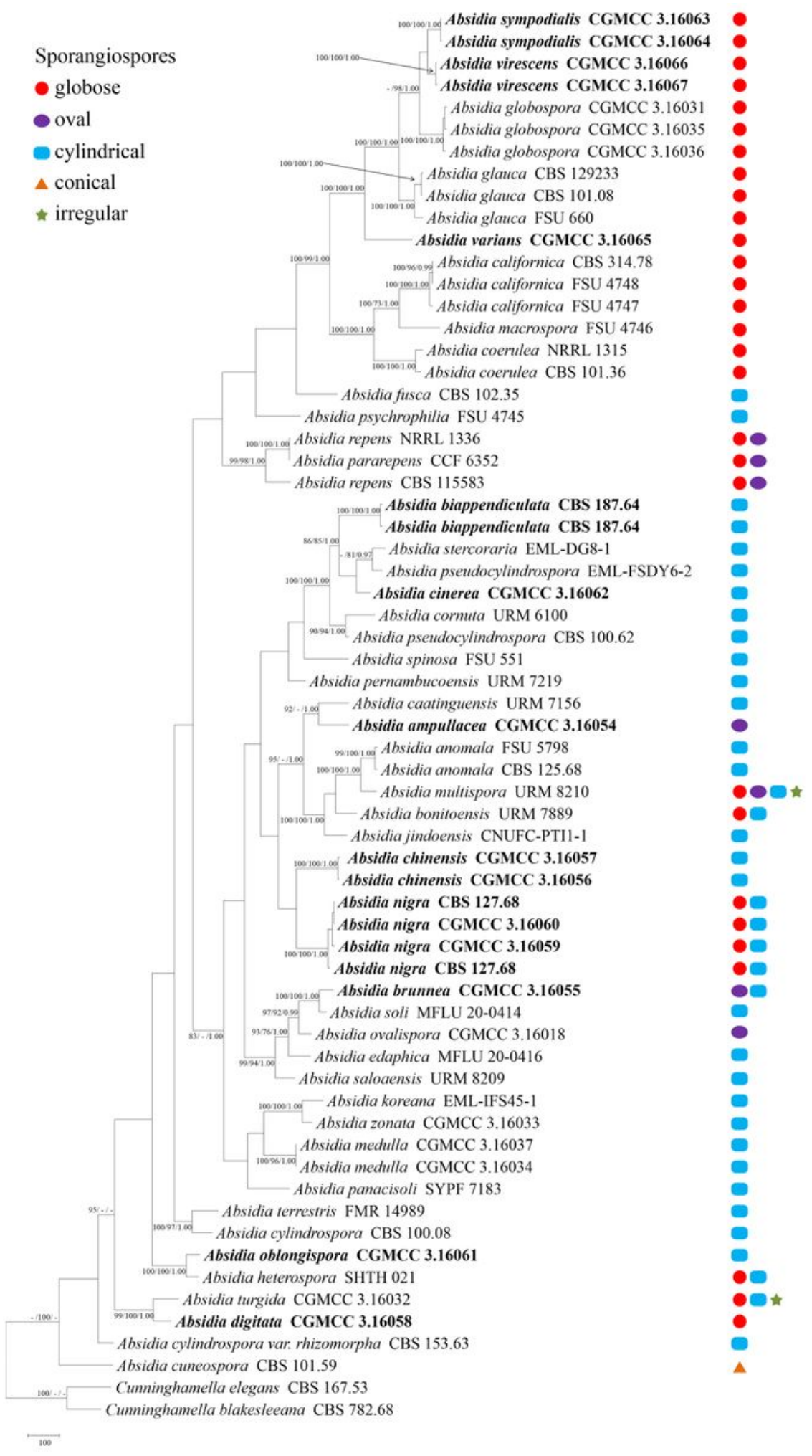

Figure 1

The Maximum Parsimony strict consensus tree illustrating the phylogeny of nine new species and two new combinations of Absidia in Cunninghamellaceae based on multi-gene sequences. Cunninghamella elegans and C. blakesleeana serve as outgroups. Branches are labeled with Maximum Likelihood 
bootstrap values higher than $70 \%$, Maximum Parsimony bootstrap values higher than $70 \%$ and Bayesian posterior probabilities more than 0.95 . The lower left scale represents steps. Sporangiospore shapes are annotated.
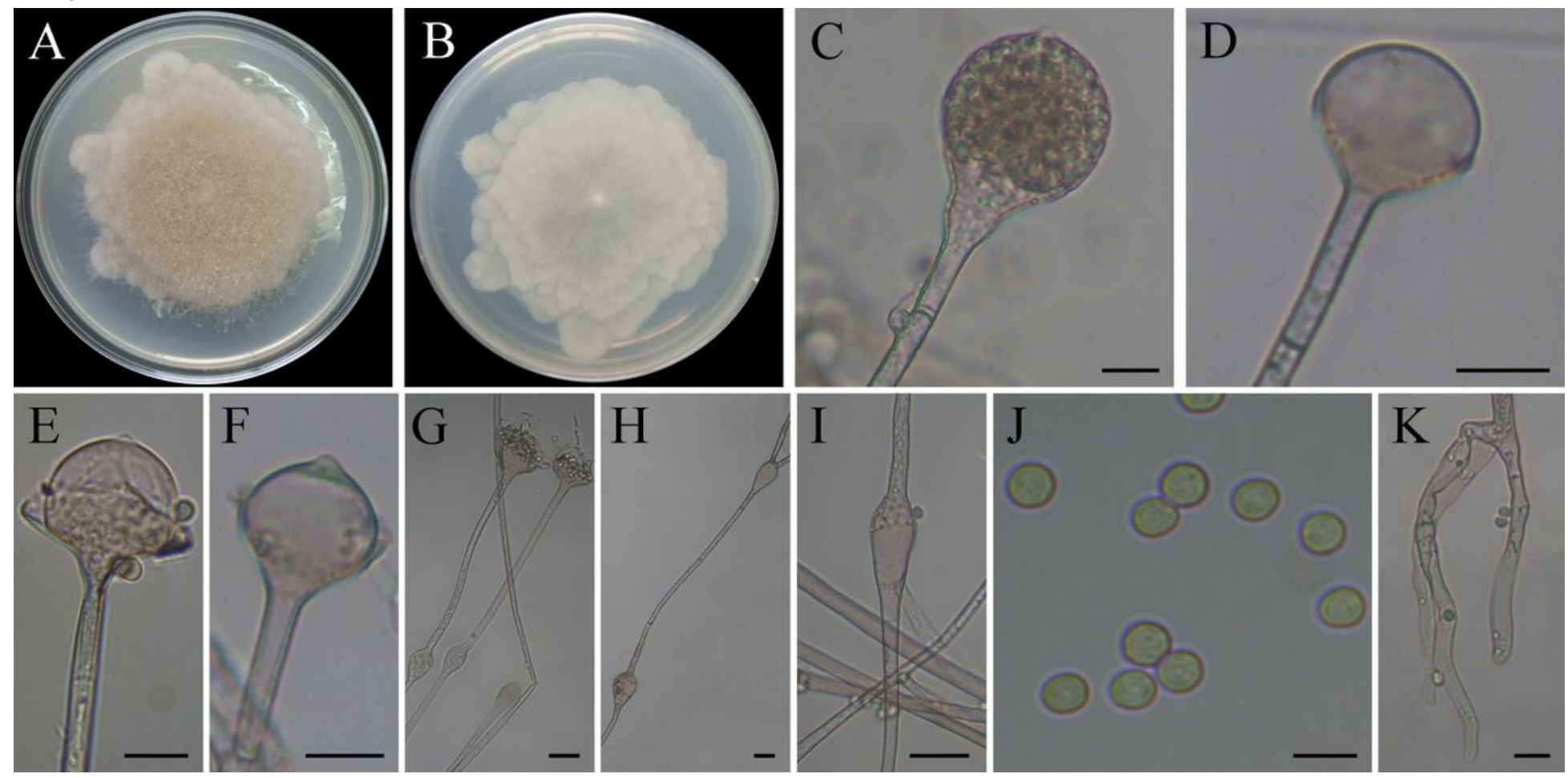

\section{Figure 2}

Absidia ampullacea CGMCC 3.16054. (A) obverse colony on MEA; (B) reverse colony on MEA; (C) sporangium; $(D-F)$ columellae; $(G-I)$ swelling on sporangiospohores and hyphae; $(J)$ sporangiospores; (K) rhizoids. Scale bars: $(C-F, K) 10 \mu m ;(G-I) 20 \mu m$; (J) $5 \mu \mathrm{m}$.
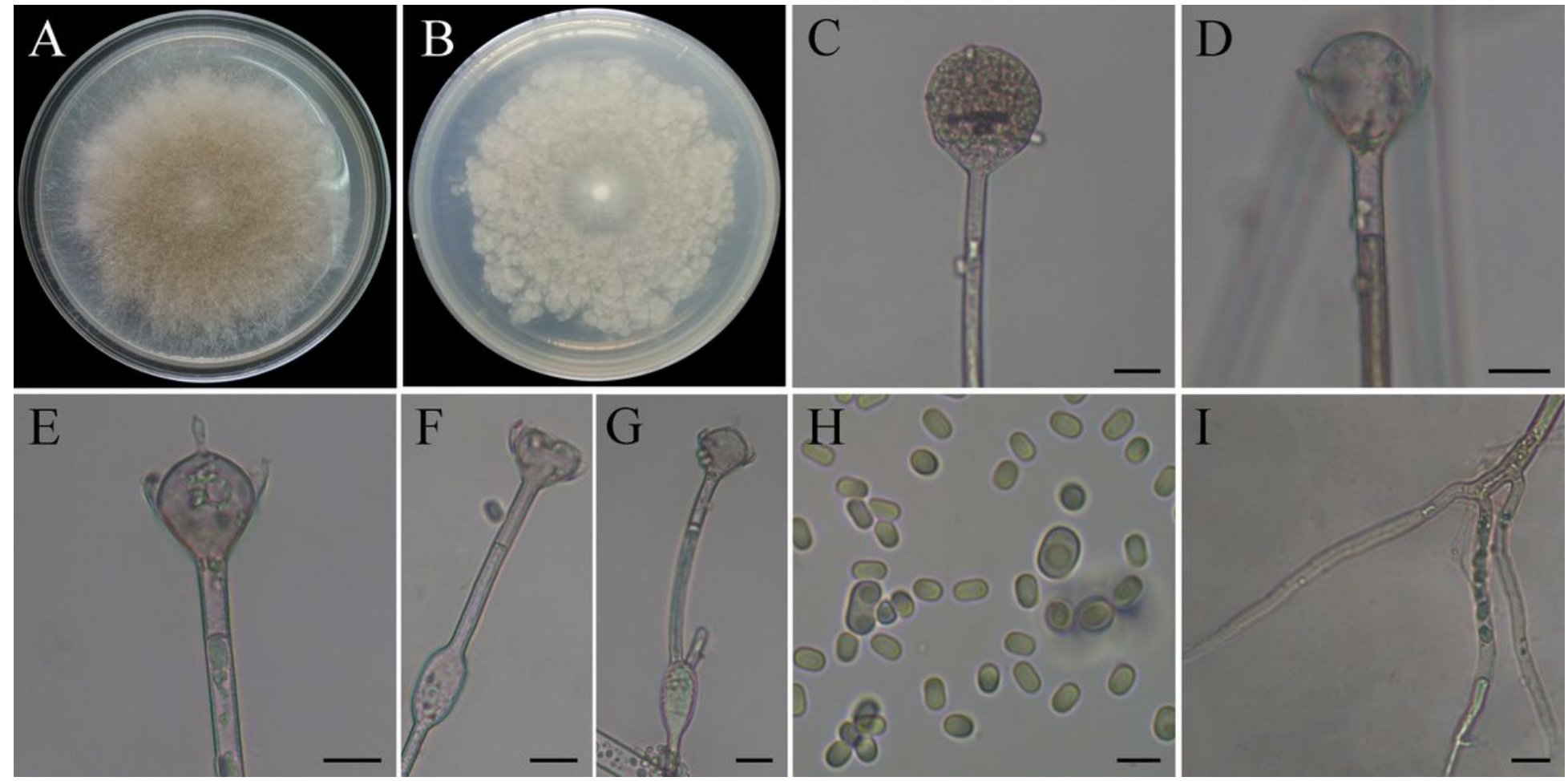


\section{Figure 3}

Absidia brunnea CGMCC 3.16055. (A) obverse colony on MEA; (B) reverse colony on MEA; (C) sporangium; $(D, E)$ columellae; $(F, G)$ swelling on sporangiospohores; $(H)$ sporangiospores; $(I)$ rhizoids. Scale bars: $(C-G, I) 10 \mu m$; (H) $5 \mu \mathrm{m}$.
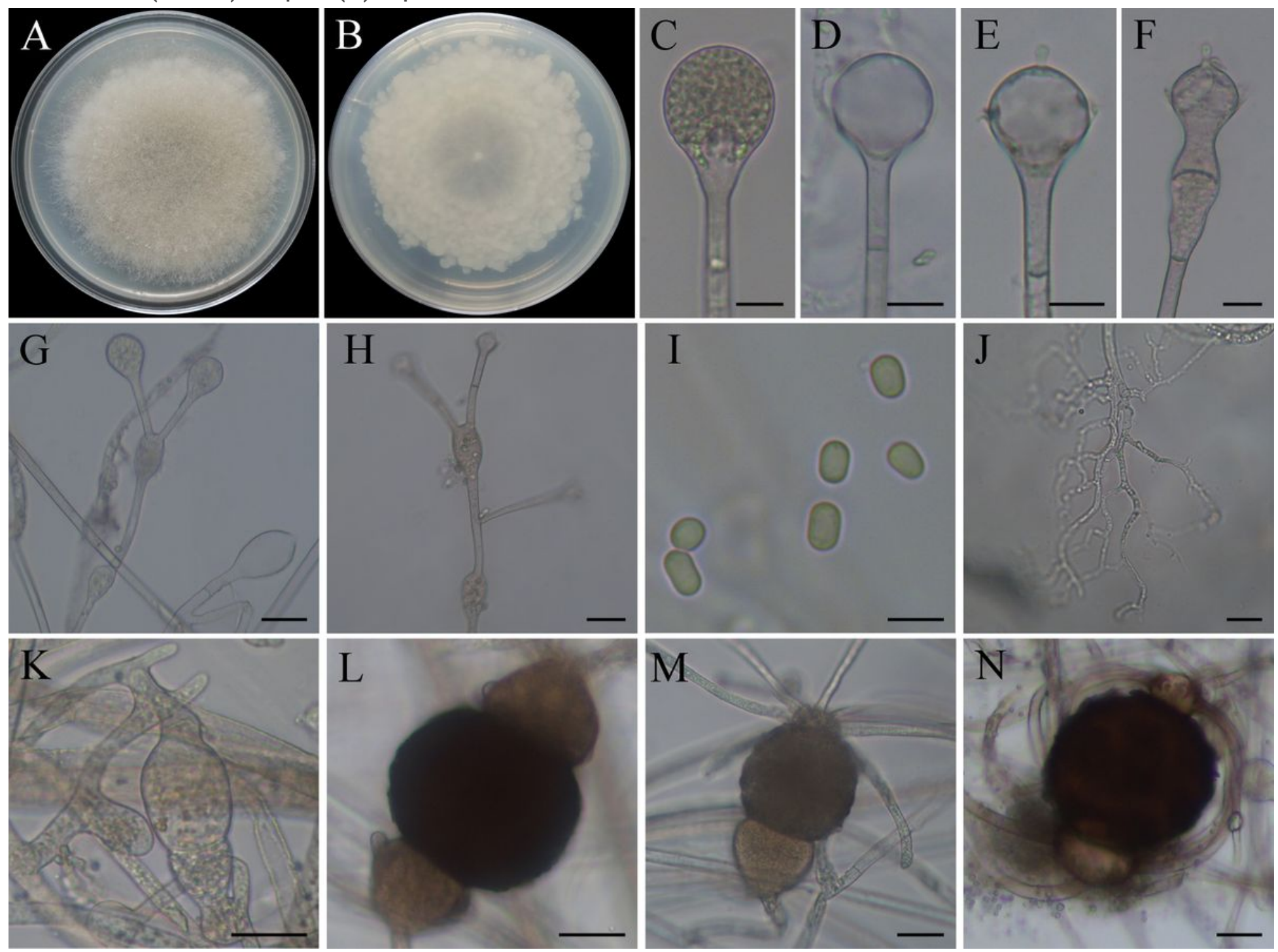

Figure 4

Absidia chinensis CGMCC 3.16056. (A) obverse colony on MEA; (B) reverse colony on MEA; (C) sporangium; (D-F) columellae; $(G, H)$ swelling on sporangiospohores; (I) sporangiospores; (J) rhizoids; $(\mathrm{K}-\mathrm{N})$ zygospores. Scale bars: $(\mathrm{C}-\mathrm{F}, \mathrm{J}-\mathrm{N}) 10 \mu \mathrm{m} ;(\mathrm{G}, \mathrm{H}) 20 \mu \mathrm{m}$; (I) $5 \mu \mathrm{m}$. 

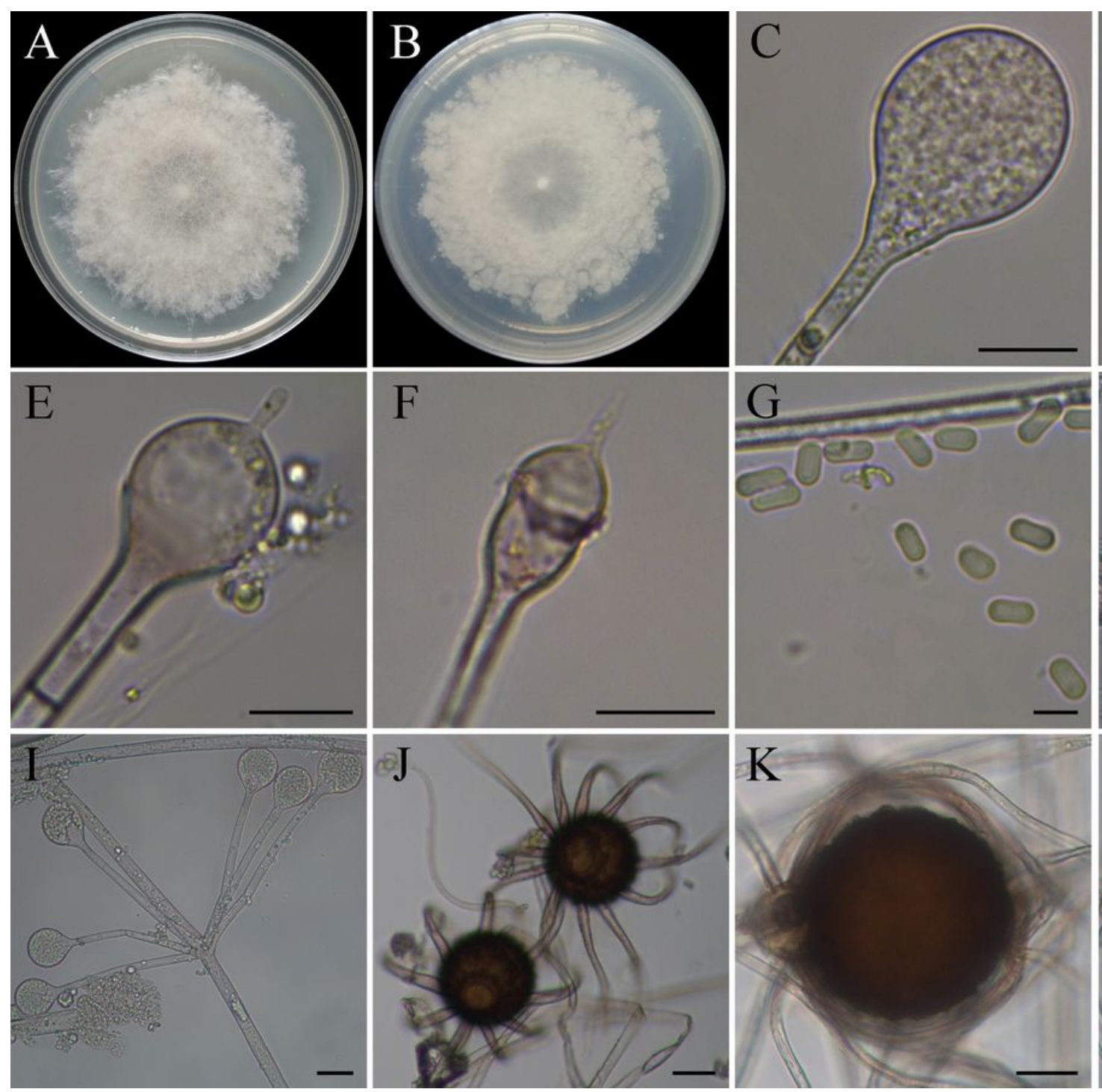
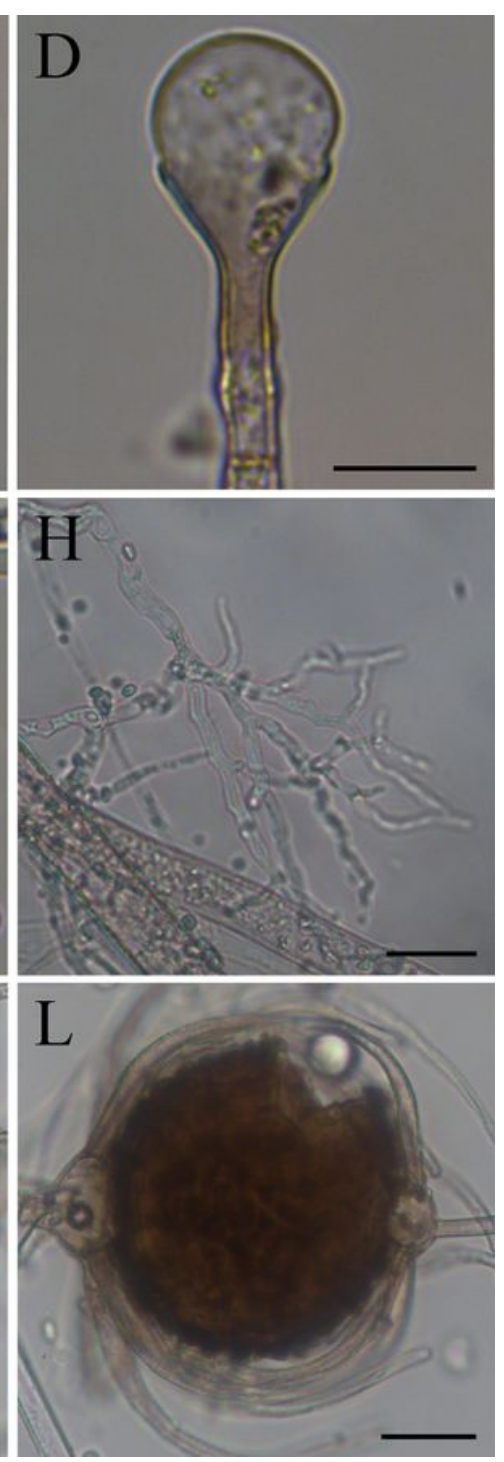

\section{Figure 5}

Absidia cinerea CGMCC 3.16062. (A) obverse colony on MEA; (B) reverse colony on MEA; (C) sporangium; $(D-F)$ columellae; $(G)$ sporangiospores; $(H)$ rhizoids $(I)$ sporangiophores in whorls; $(J-L)$ zygospores.

Scale bars: (C-F) $10 \mu \mathrm{m}$; (G) $5 \mu \mathrm{m}$; (H-L) $20 \mu \mathrm{m}$. 

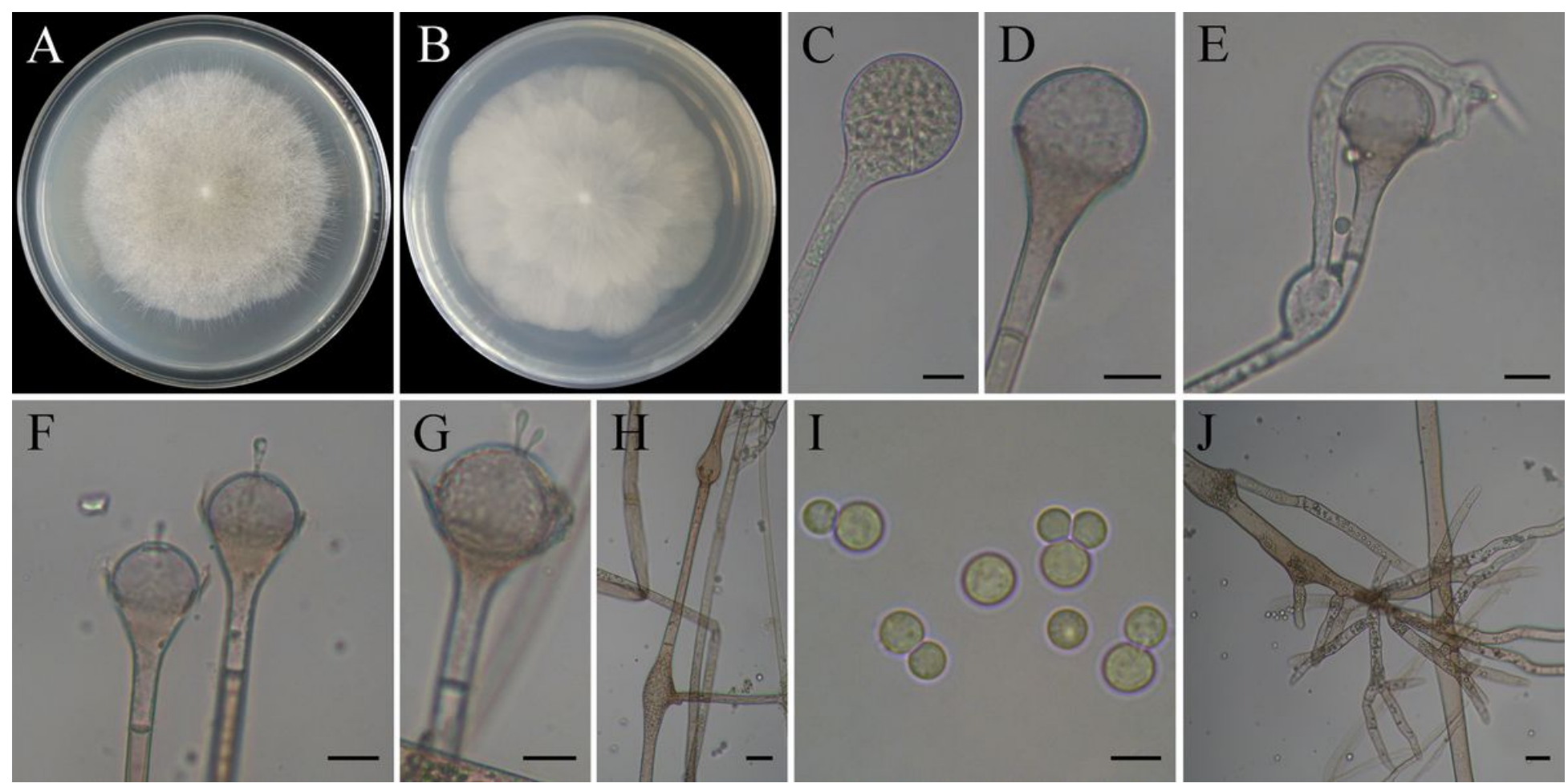

Figure 6

Absidia digitata CGMCC 3.16058. (A) obverse colony on MEA; (B) reverse colony on MEA; (C) sporangium; $(D-G)$ columellae; $(H)$ swelling on hyphae; $(I)$ sporangiospores; $(J)$ rhizoids. Scale bars: $(C-G) 10 \mu m ;(H$, J) $20 \mu \mathrm{m}$; (I) $5 \mu \mathrm{m}$.
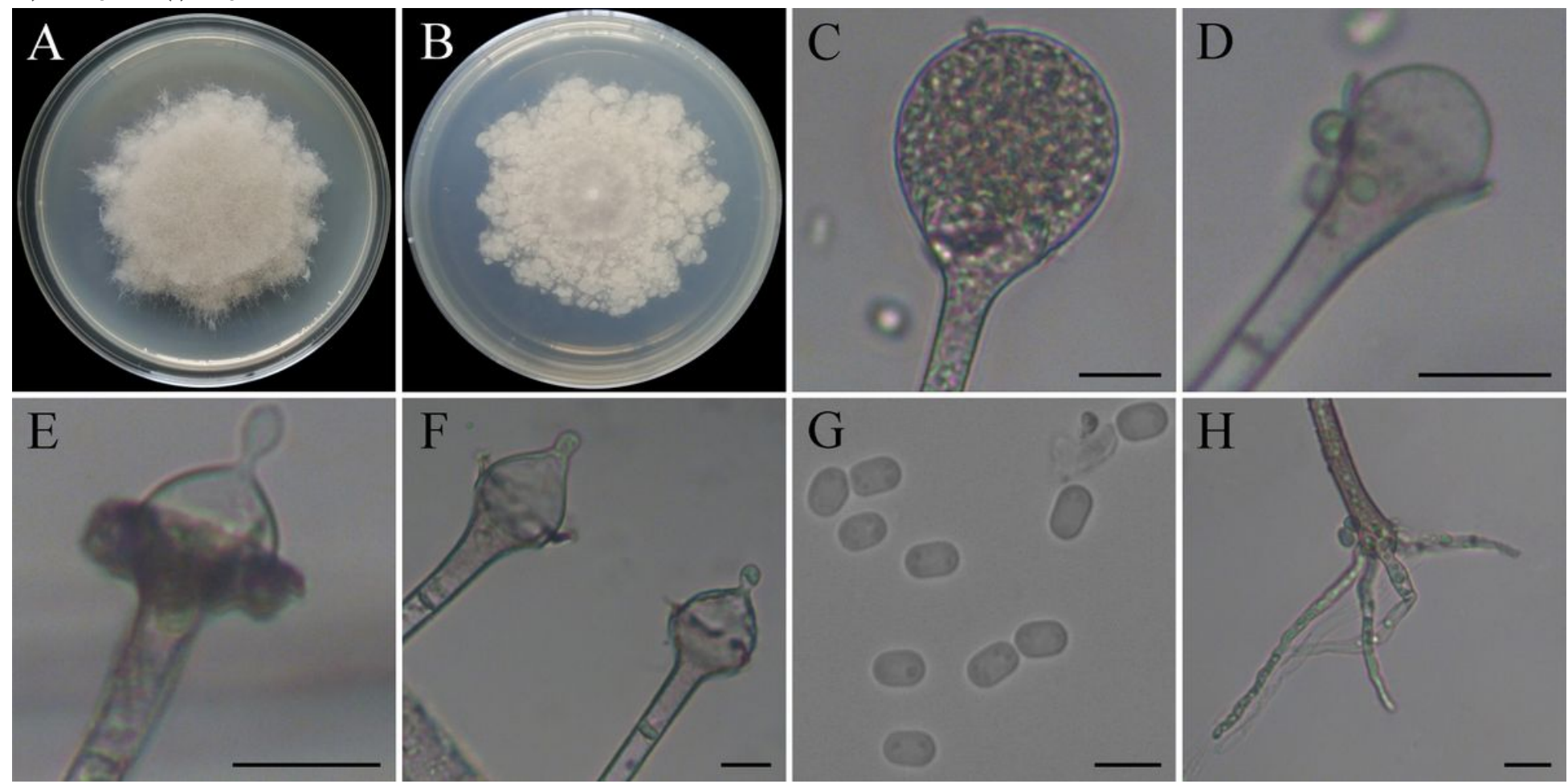

Figure 7 
Absidia oblongispora CGMCC 3.16061. (A) obverse colony on MEA; (B) reverse colony on MEA; (C)

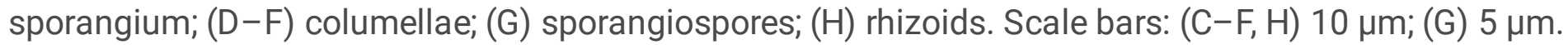
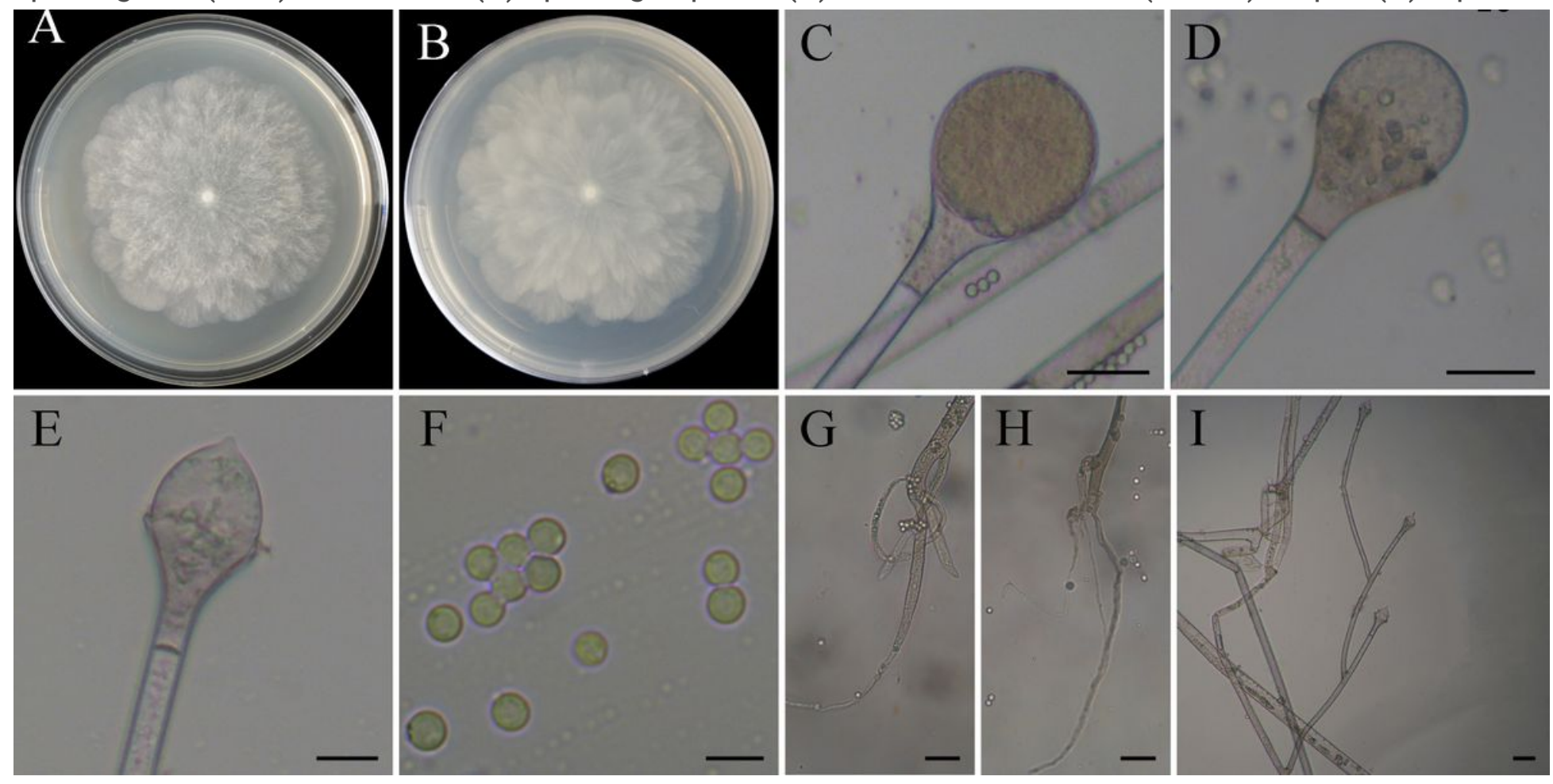

\section{Figure 8}

Absidia sympodialis CGMCC 3.16064. (A) obverse colony on MEA; (B) reverse colony on MEA; (C) sporangium; (D, E) columellae; (F) sporangiospores; (G, H) rhizoids; (I) sympodial sporangiospores. Scale bars: $(C-E, G-I) 20 \mu m ;(F) 5 \mu m$. 


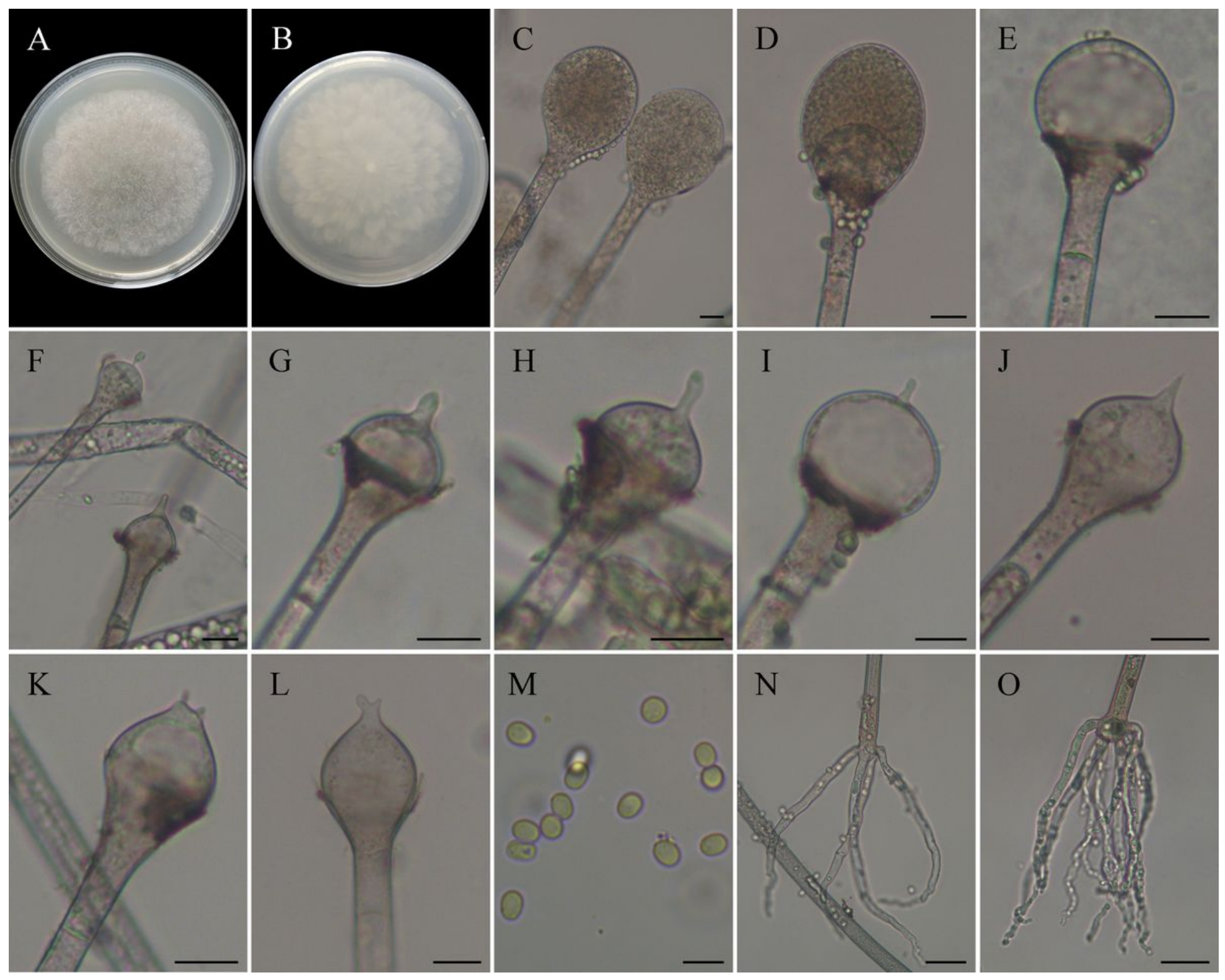

Figure 9

Absidia varians CGMCC 3.16065. (A) obverse colony on MEA; (B) reverse colony on MEA; (C, D) sporangia; $(E-L)$ columellae; $(M)$ sporangiospores; $(N, 0)$ rhizoids. Scale bars: $(C-L) 10 \mu m ;(M) 5 \mu m ;(N$, 0) $20 \mu \mathrm{m}$. 

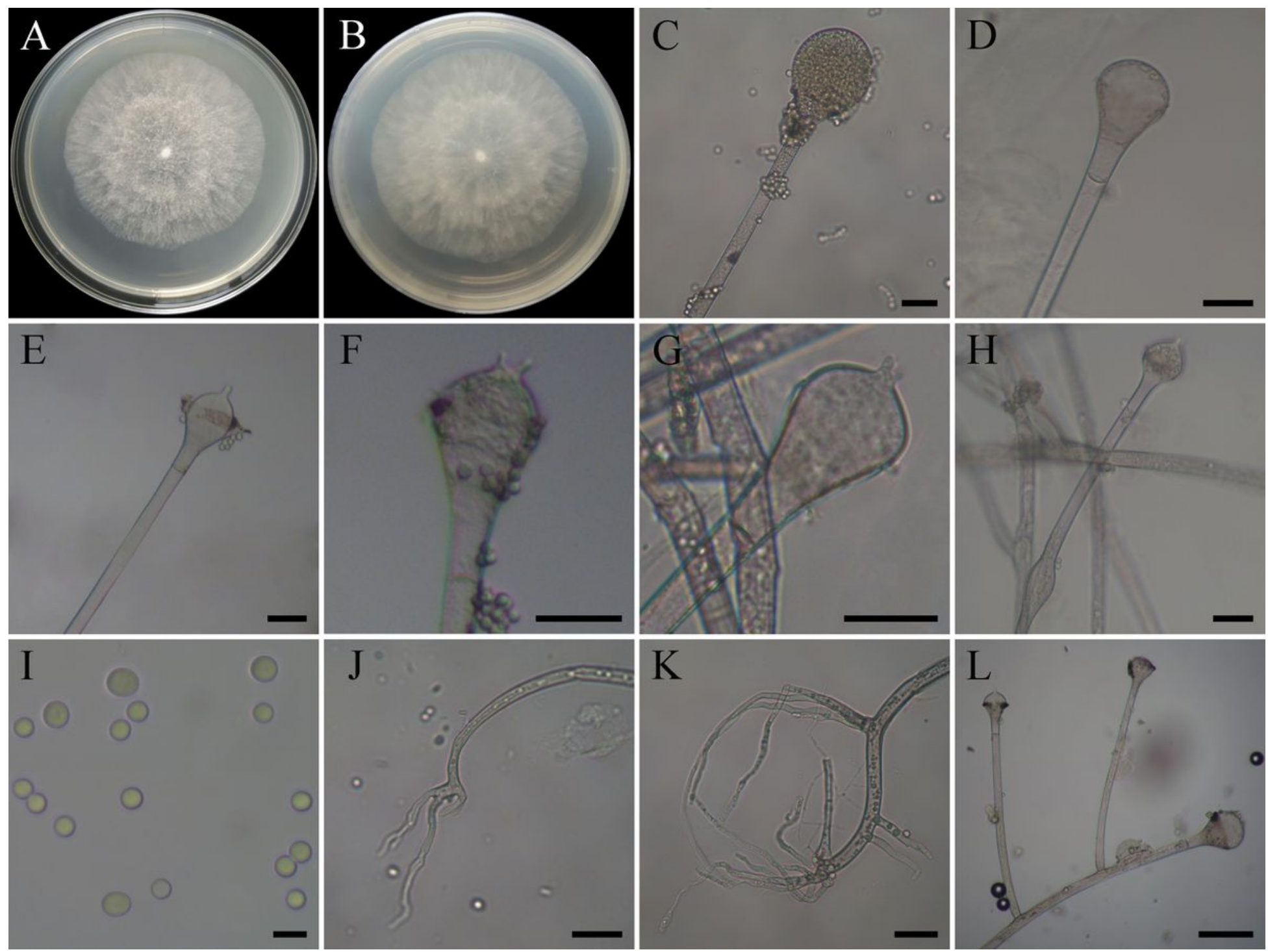

Figure 10

Absidia virescens CGMCC 3.16067. (A) obverse colony on MEA; (B) reverse colony on MEA; (C) sporangium; (D-G) columellae; $(\mathrm{H})$ swelling on sporangiospohores; (I) sporangiospores; $(\mathrm{J}, \mathrm{K})$ rhizoids; (L) monopodial sporangiospohores. Scale bars: (C-H, J-K) $20 \mu \mathrm{m}$; (I) $5 \mu \mathrm{m}$; (L) $50 \mu \mathrm{m}$. 

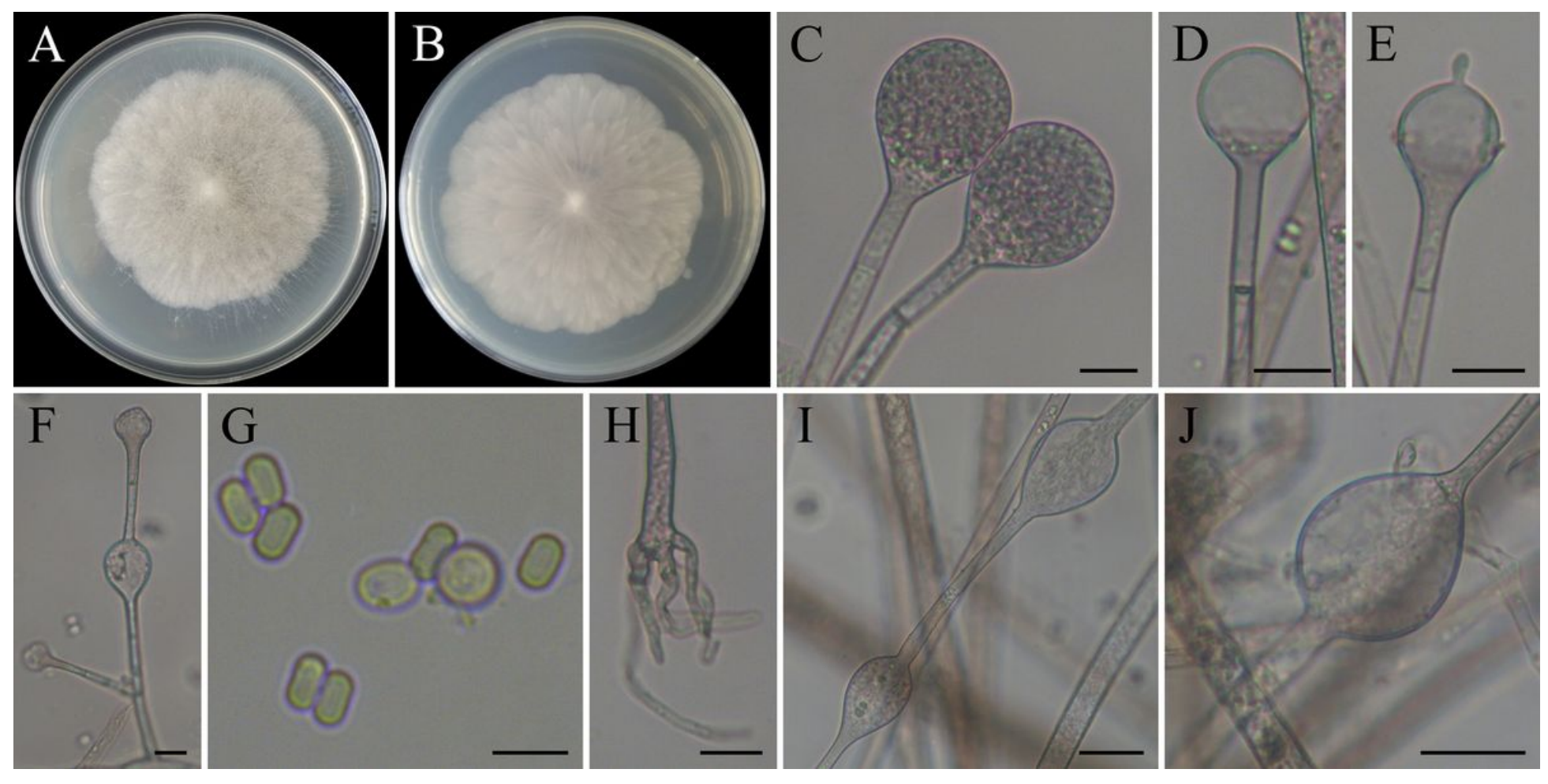

\section{Figure 11}

Absidia nigra CGMCC 3.16059. (A) obverse colony on MEA; (B) reverse colony on MEA; (C) sporangia; (D, E) columellae; (F) swelling on sporangiospohores; $(G)$ sporangiospores; $(H)$ rhizoids $(I, J)$ swollen hyphae. Scale bars: (C-F, H) $10 \mu \mathrm{m}$; (G) $5 \mu \mathrm{m}$; (I, J) $20 \mu \mathrm{m}$. 

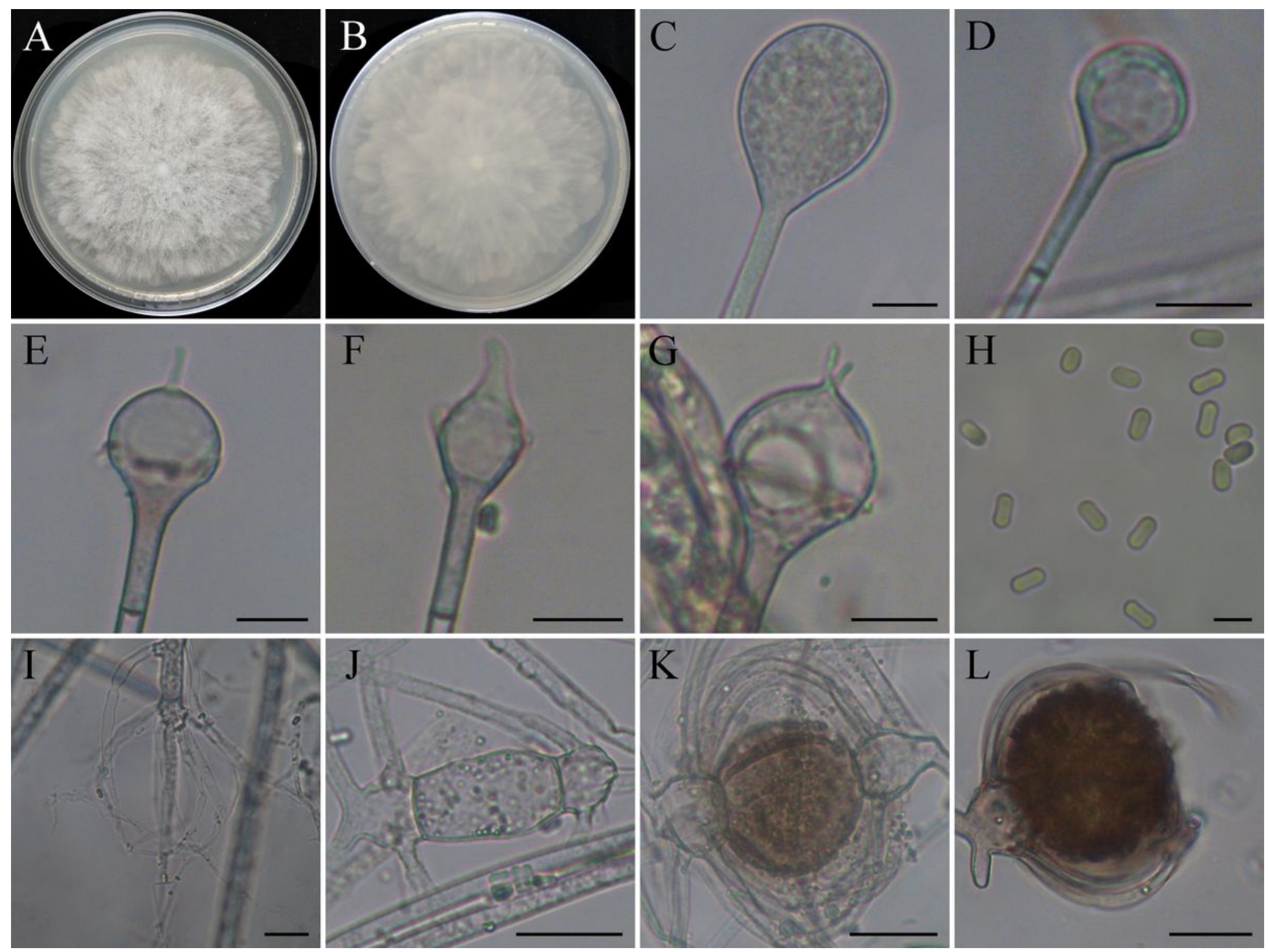

Figure 12

Absidia biappendiculata CBS 187.64. (A) obverse colony on MEA; (B) reverse colony on MEA; (C) sporangium; (D-G) columellae; $(H)$ sporangiospores; (I) rhizoids; $(K-L)$ zygospores. Scale bars: $(C-G) 10$ $\mu \mathrm{m} ;(\mathrm{H}) 5 \mu \mathrm{m} ;(\mathrm{I}-\mathrm{L}) 20 \mu \mathrm{m}$. 


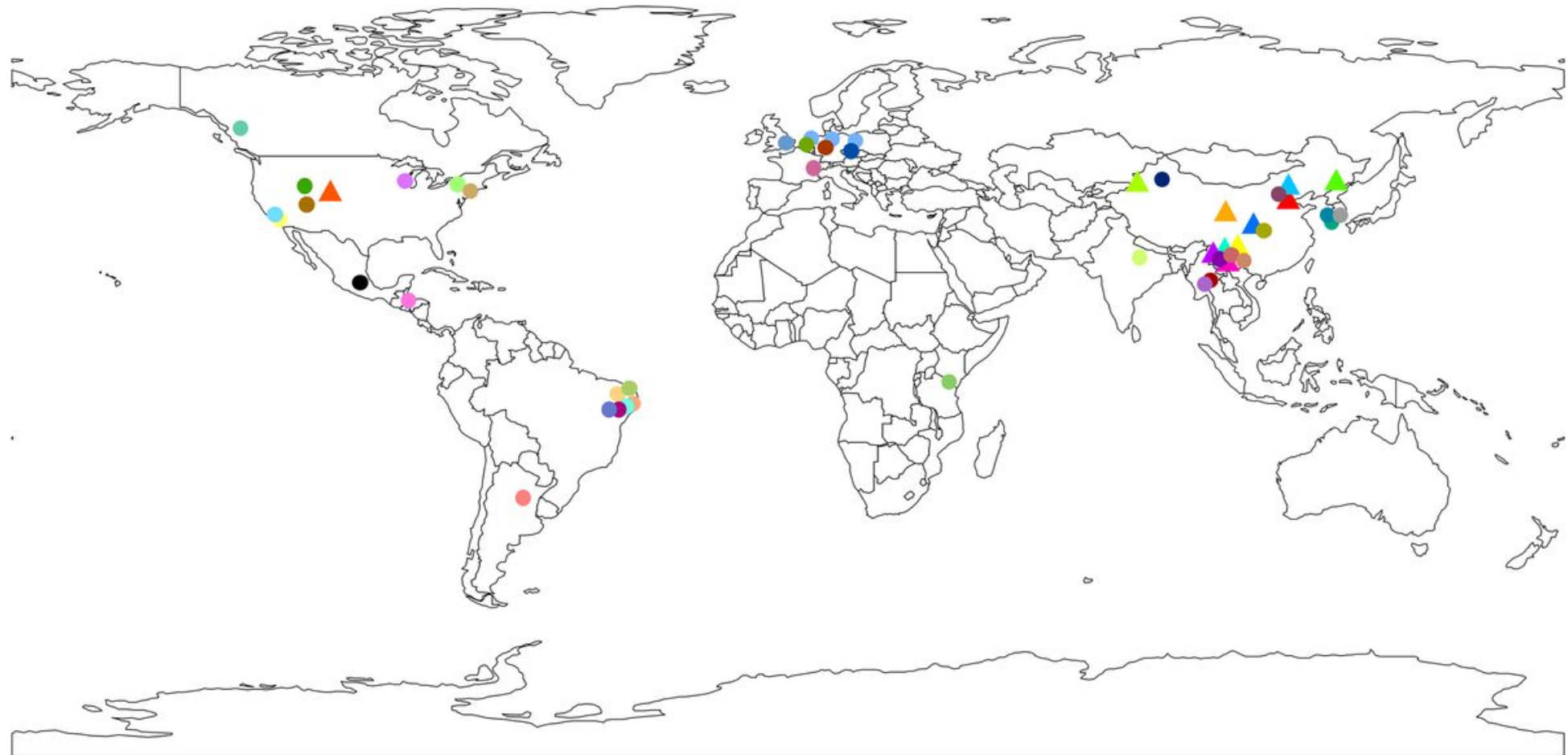
- A. ampullacea
A A. biappendiculata
A A. brunnea
A. chinensis
$\Delta$ A. cinerea
A A. digitata
4 A. nigra
A A. oblongispora
A A. sympodialis
A A. virescens

- A. anomala

- A. cylindrospora var. nigra

- A. korean

- A. psychrophilia

A. bonitoensis

A. cylindrospora var. rhizomorpha

- A. macrospor

- A. repens

A. caatinguensis

- A. edaphica

- A. medulla

- A. fusca

A. clavata

- A. glauca

- A. multispora

- A. saloaensis

A. coerulea

- A. globospora

- A. ovalispora

- A. soli

A. cornuta

- A. cuneospora

- A. heterospora

- A.panacisoli

- A. spinosa

- A. cylindrospora

- A. idahoensis

- A. pararepens

- A. pernambucoensis

- A. jindoensis

- A. pseudocylindrospora

- A. stercoraria

- A. terrestris

- A. turgida

- A. zonata

\section{Figure 13}

The geographic distribution of Absida types except for A. glauca and A. cylindrospora (neither is available). Triangles represent nine new species and two combinations proposed in this study. Circles represent previously reported species. 